Algebraic 83 Geometric $\mathcal{T}_{\text {opology }}$

Volume 5 (2005) 923-964

Published: 5 August 2005

ATG

\title{
Conjugation spaces
}

\author{
Jean-Claude Hausmann \\ TARA HOLM \\ Volker Puppe
}

\begin{abstract}
There are classical examples of spaces $X$ with an involution $\tau$ whose mod2-comhomology ring resembles that of their fixed point set $X^{\tau}$ : there is a ring isomorphism $\kappa: H^{2 *}(X) \approx H^{*}\left(X^{\tau}\right)$. Such examples include complex Grassmannians, toric manifolds, polygon spaces. In this paper, we show that the ring isomorphism $\kappa$ is part of an interesting structure in equivariant cohomology called an $H^{*}$-frame. An $H^{*}$-frame, if it exists, is natural and unique. A space with involution admitting an $\mathrm{H}^{*}$-frame is called a conjugation space. Many examples of conjugation spaces are constructed, for instance by successive adjunctions of cells homeomorphic to a disk in $\mathbb{C}^{k}$ with the complex conjugation. A compact symplectic manifold, with an anti-symplectic involution compatible with a Hamiltonian action of a torus $T$, is a conjugation space, provided $X^{T}$ is itself a conjugation space. This includes the co-adjoint orbits of any semi-simple compact Lie group, equipped with the Chevalley involution. We also study conjugateequivariant complex vector bundles ("real bundles" in the sense of Atiyah) over a conjugation space and show that the isomorphism $\kappa$ maps the Chern classes onto the Stiefel-Whitney classes of the fixed bundle.
\end{abstract}

AMS Classification 55N91, 55M35; 53D05, 57R22

Keywords Cohomology rings, equivariant cohomology, spaces with involution, real spaces

\section{Introduction}

In this article, we study topological spaces equipped with a continuous involution. We are motivated by the example of the complex Grassmannian $\operatorname{Gr}\left(k, \mathbb{C}^{n}\right)$ of complex $k$-vector subspaces of $\mathbb{C}^{n}(n \leq \infty)$, with the involution complex conjugation. The fixed point set of this involution is the real Grassmannian $\operatorname{Gr}\left(k, \mathbb{R}^{n}\right)$. It is well known that there is a ring isomorphism $\kappa: H^{2 *}\left(\operatorname{Gr}\left(k, \mathbb{C}^{n}\right)\right) \approx H^{*}\left(\operatorname{Gr}\left(k, \mathbb{R}^{n}\right)\right)$ in cohomology (with $\mathbb{Z}_{2}$-coefficients) dividing the degree of a class in half. Other such isomorphisms have been found for 
natural involutions on smooth toric manifolds [7] and polygon spaces [13, $\S 9$ ]. The significance of this isomorphism property was first discussed by A. Borel and A. Haefliger [6] in the framework of analytic geometry. For more recent consideration in the context of real algebraic varieties, see [28, 29].

The goal of this paper is to show that, for the above examples and many others, the ring isomorphism $\kappa$ is part of an interesting structure in equivariant cohomology. We will use $H^{*}$ to denote singular cohomology, taken with $\mathbb{Z}_{2}$ coefficients. For a group $C$, we will let $H_{C}^{*}$ denote $C$-equivariant cohomology with $\mathbb{Z}_{2}$ coefficients, using the Borel construction [5]. Let $\tau$ be a (continuous) involution on a topological space $X$. We view this as an action of the cyclic group of order two, $C=\{I, \tau\}$. Let $\rho: H_{C}^{2 *}(X) \rightarrow H^{2 *}(X)$ and $r: H_{C}^{*}(X) \rightarrow H_{C}^{*}\left(X^{\tau}\right)$ be the restriction homomorphisms in cohomology. We use that $H_{C}^{*}\left(X^{\tau}\right)=H^{*}\left(X^{\tau} \times B C\right)$ is naturally isomorphic to the polynomial ring $H^{*}\left(X^{\tau}\right)[u]$ where $u$ is of degree one. Suppose that $H^{\text {odd }}(X)=0$. A cohomology frame or $H^{*}$-frame for $(X, \tau)$ is a pair $(\kappa, \sigma)$, where

(a) $\kappa: H^{2 *}(X) \rightarrow H^{*}\left(X^{\tau}\right)$ is an additive isomorphism dividing the degrees in half; and

(b) $\sigma: H^{2 *}(X) \rightarrow H_{C}^{2 *}(X)$ is an additive section of $\rho$.

In addition, $\kappa$ and $\sigma$ must satisfy the conjugation equation

$$
r \circ \sigma(a)=\kappa(a) u^{m}+\ell t_{m},
$$

for all $a \in H^{2 m}(X)$ and all $m \in \mathbb{N}$, where $\ell t_{m}$ denotes some polynomial in the variable $u$ of degree less than $m$.

An involution admitting a $H^{*}$-frame is called a conjugation and a space together with a conjugation is called a conjugation space. Required to be only additive maps, $\kappa$ and $\sigma$ are often easy to construct degree by degree. But we will show in the "multiplicativity theorem" in Section 3 that in fact $\sigma$ and $\kappa$ are ring homomorphisms. Moreover, given a $C$-equivariant map $f: Y \rightarrow X$ between spaces with involution, along with $H^{*}$-frames $\left(\sigma_{X}, \kappa_{X}\right)$ and $\left(\sigma_{Y}, \kappa_{Y}\right)$, we have $H_{C}^{*} f \circ \sigma_{X}=\sigma_{Y} \circ H^{*} f$ and $H^{*} f^{\tau} \circ \kappa_{X}=\kappa_{Y} \circ H^{*} f$. In particular, the $H^{*}$-frame for a conjugation is unique.

As an example of a conjugation space, one has the complex projective space $\mathbb{C} P^{k}(k \leq \infty)$, with the complex conjugation as involution. If $a$ is the generator of $H^{2}\left(\mathbb{C} P^{k}\right)$ and $b=\kappa(a)$ that of $H^{1}\left(\mathbb{R} P^{k}\right)$, we will see that the conjugation equation has the form $r \circ \sigma\left(a^{m}\right)=\left(b u+b^{2}\right)^{m}$ (Example 3.7).

The complex projective spaces are particular cases of spherical conjugation complexes, which constitute our main class of examples. A spherical conjugation complex is a space (with involution) obtained from the empty set by 
countably many successive adjunction of collections of conjugation cells. A conjugation cell (of dimension $2 k$ ) is a space with involution which is equivariantly homeomorphic to the closed disk of radius 1 in $\mathbb{R}^{2 k}$, equipped with a linear involution with exactly $k$ eigenvalues equal to -1 . At each step, the collection of conjugation cells consists of cells of the same dimension but, as in 11], the adjective "spherical" is a warning that these dimensions do not need to be increasing. We prove that every spherical conjugation complex is a conjugation space. There are many examples of these; for instance, there are infinitely many $C$-equivariant homotopy types of spherical conjugation complexes with three conjugation cells, one each in dimensions 0,2 and 4 . We prove that for a $C$-equivariant fibration (with a compact Lie group as structure group) whose fiber is a conjugation space and whose base is a spherical conjugation complex, then its total space is a conjugation space.

Schubert cells for Grassmannians are conjugation cells, so these spaces are spherical conjugation complexes and therefore conjugation spaces. This generalizes in the following way. Let $X$ be a space together with an involution $\tau$ and a continuous action of a torus $T$. We say that $\tau$ is compatible with this torus action if $\tau(g \cdot x)=g^{-1} \cdot \tau(x)$ for all $g \in T$ and $x \in X$. It follows that $\tau$ induces an involution on the fixed point set $X^{T}$ and an action of the 2-torus $T_{2}$ (the elements of order 2) of $T$ on $X^{\tau}$. We are particularly interested in the case when $X$ is a compact symplectic manifold for which the torus action is Hamiltonian and the compatible involution is smooth and anti-symplectic. Using a Morse-Bott function obtained from the moment map for the $T$-action, we prove that if $X^{T}$ is a conjugation space (respectively a spherical conjugation complex), then $X$ is a conjugation space (respectively a spherical conjugation complex). In addition, we prove that the involution induced on the Borel construction $X_{T}$ is a conjugation. The relevant isomorphism $\bar{\kappa}$ takes the form of a natural ring isomorphism

$$
\bar{\kappa}: H_{T}^{2 *}(X) \stackrel{\approx}{\longrightarrow} H_{T_{2}}^{*}\left(X^{\tau}\right) .
$$

Examples of such Hamiltonian spaces include co-adjoint orbits of any semisimple compact Lie group, with the Chevalley involution, smooth toric manifolds and polygon spaces. Consequently, these examples are spherical conjugation complexes. For the co-adjoint orbits of $S U(n)$ this was proved earlier by C. Schmid 24] and D. Biss, V. Guillemin and the second author [4. The category of conjugation spaces is closed under various operations, including direct products, connected sums and, under some hypothesis, under symplectic reduction (generalizing [9]; see Subsection [.4). This yields more examples of conjugation spaces. 
Over spaces with involution, it is natural to study conjugate equivariant bundles, identical to the "real bundles" introduced by Atiyah [2]. These are complex vector bundles $\eta=(E \stackrel{p}{\longrightarrow} X)$ together with an involution $\hat{\tau}$ on $E$, which covers $\tau$ and is conjugate linear on each fiber. Then $E^{\hat{\tau}}$ is a real bundle $\eta^{\tau}$ over $X^{\tau}$. In Section 6. we prove several results on conjugate equivariant bundles, among them that if $\eta=(E \stackrel{p}{\longrightarrow} X)$ is a conjugate equivariant bundle over a conjugation space, then the Thom space is a conjugation space. These results are used in the proof of the aforementioned theorems in symplectic geometry. Finally, when the basis of a conjugate equivariant bundle is a spherical conjugation complex, we prove that $\kappa(c(\eta))=w\left(\eta^{\tau}\right)$, where $c()$ denotes the (mod 2) total Chern class and $w()$ the total Stiefel-Whitney class.

Acknowledgments Anton Alekseev gave us precious suggestions for Subsection 8.3 on the Chevalley involution on coadjoint orbits. Conversations with Matthias Franz were very helpful. The first two authors are also grateful to Sue Tolman for pointing out a gap in an earlier stage of this project. Finally, we thank Martin Olbermann for useful observations.

The three authors thank the Swiss National Funds for Scientific Research for its support. The second author was supported in part by a National Science Foundation Postdoctoral Fellowship.

\section{Preliminaries}

Let $\tau$ be a (continuous) involution on a space $X$. This gives rise to a continuous action of the cyclic group $C=\{1, \tau\}$ of order 2 . The real locus $X^{\tau} \subset X$ is the subspace of $X$ formed by the elements which are fixed by $\tau$.

Unless otherwise specified, all the cohomology groups are taken with $\mathbb{Z}_{2}$-coefficients. A pair $(X, Y)$ is an even cohomology pair if $H^{\text {odd }}(X, Y)=0$; a space $X$ is an even cohomology space if $(X, \emptyset)$ is an even cohomology pair.

2.1 Let $R$ be the graded ring $R=H^{*}(B C)=H_{C}^{*}(p t)=\mathbb{Z}_{2}[u]$, where $u$ is in degree 1 . We denote by $R^{e v}$ the subring of $R$ of elements of even degree.

As $C$ acts trivially on the real locus $X^{\tau}$, there is a natural identification $E C \times_{C}$ $X^{\tau} \stackrel{\approx}{\rightarrow} B C \times X^{\tau}$. The Künneth formula provides a ring isomorphism $R \otimes$ $H^{*}\left(X^{\tau}, Y^{\tau}\right) \stackrel{\approx}{\rightarrow} H_{C}^{*}\left(X^{\tau}, Y^{\tau}\right)$ and $R \otimes H^{*}\left(X^{\tau}, Y^{\tau}\right)$ is naturally isomorphic to the polynomial ring $H^{*}\left(X^{\tau}, Y^{\tau}\right)[u]$. We shall thus often use the "Künneth isomorphism" $K: H^{*}\left(X^{\tau}, Y^{\tau}\right)[u] \stackrel{\approx}{\longrightarrow} H_{C}^{*}\left(X^{\tau}, Y^{\tau}\right)$ to identify these two rings. The naturality of $K$ gives the following: 
Lemma 2.2 Let $f:\left(X_{2}, Y_{2}\right) \rightarrow\left(X_{1}, Y_{1}\right)$ be a continuous $C$-equivariant map between pairs with involution. Let $f^{\tau}:\left(X_{2}^{\tau}, Y_{2}^{\tau}\right) \rightarrow\left(X_{1}^{\tau}, Y_{1}^{\tau}\right)$ be the restriction of $f$ to the fixed point sets. Then, the following diagram

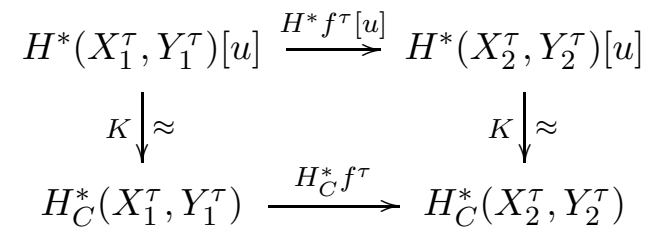

is commutative, where $H^{*} f^{\tau}[u]$ is the polynomial extension of $H^{*} f^{\tau}$.

2.3 Equivariant formality Let $X$ be a space with an involution $\tau$ and let $Y$ be a $\tau$-invariant subspace of $X$ (i.e. $\tau(Y)=Y$ ). Following 10, we say that the pair $(X, Y)$ is equivariantly formal (over $\left.\mathbb{Z}_{2}\right)$ if the map $(X, Y) \rightarrow$ $\left(E C \times_{C} X, E C \times_{C} Y\right)$ is totally non-homologous to zero. That is, the restriction homomorphism $\rho: H_{C}^{*}(X, Y) \rightarrow H^{*}(X, Y)$ is surjective. A space $X$ with involution is equivariantly formal if the pair $(X, \emptyset)$ is equivariantly formal.

If $(X, Y)$ is equivariantly formal, one can choose, for each $k \in \mathbb{N}$, a $\mathbb{Z}_{2}$-linear map $\sigma: H^{k}(X, Y) \rightarrow H_{C}^{k}(X, Y)$ such that $\rho \circ \sigma=\mathrm{id}$. This gives an additive section $\sigma: H^{*}(X, Y) \rightarrow H_{C}^{*}(X, Y)$ of $\rho$ which gives rise to a map

$$
\hat{\sigma}: H^{*}(X, Y)[u] \rightarrow H_{C}^{*}(X, Y) \text {. }
$$

As in 2.1] we use the ring isomorphism $H^{*}(X, Y) \otimes R \stackrel{\approx}{\longrightarrow} H^{*}(X, Y)[u]$. As $H^{*}(B C)=R$, the Leray-Hirsch theorem (see e.g. [20, Theorem 5.10]) then implies that $\hat{\sigma}$ is an isomorphism of $R$-modules. But $\hat{\sigma}$ is not in general an isomorphism of rings. This is the case if and only if the section $\sigma$ is a ring homomorphism but such ring-sections do not usually exist.

\section{Conjugation pairs and spaces}

\subsection{Definitions and the multiplicativity theorem}

Let $\tau$ be an involution on a space $X$ and let $Y$ be a $\tau$-invariant subspace of $X$. Let $\rho: H_{C}^{2 *}(X, Y) \rightarrow H^{2 *}(X, Y)$ and $r: H_{C}^{*}(X, Y) \rightarrow H_{C}^{*}\left(X^{\tau}, Y^{\tau}\right)$ be the restriction homomorphisms. A cohomology frame or $H^{*}$-frame for $(X, Y)$ is a pair $(\kappa, \sigma)$, where

(a) $\kappa: H^{2 *}(X, Y) \rightarrow H^{*}\left(X^{\tau}, Y^{\tau}\right)$ is an additive isomorphism dividing the degrees in half; and 
(b) $\sigma: H^{2 *}(X, Y) \rightarrow H_{C}^{2 *}(X, Y)$ is an additive section of $\rho$.

Moreover, $\kappa$ and $\sigma$ must satisfy the conjugation equation

$$
r \circ \sigma(a)=\kappa(a) u^{m}+\ell t_{m}
$$

for all $a \in H^{2 m}(X)$ and all $m \in \mathbb{N}$, where $\ell_{m}$ denotes any polynomial in the variable $u$ of degree less than $m$.

An involution admitting a $H^{*}$-frame is called a conjugation. An even cohomology pair together with a conjugation is called a conjugation pair. An evencohomology space $X$ together with an involution is a conjugation space if the pair $(X, \emptyset)$ is a conjugation pair. Observe that the existence of $\sigma$ is equivalent to $(X, Y)$ being equivariantly formal. We shall see in Corollary 3.12 that the $H^{*}$-frame for a conjugation is unique.

Remark 3.1 The map $\kappa$ coincides on $H^{0}(X, Y)$ with the restriction homomorphism $\tilde{r}: H^{0}(X, Y) \rightarrow H^{0}\left(X^{\tau}, Y^{\tau}\right)$. Indeed, the following diagram

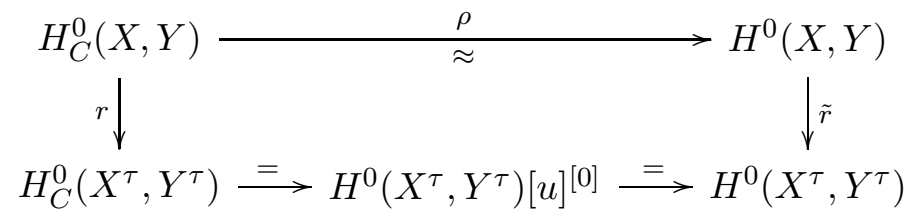

is commutative. Therefore, using Equation (3.1), one has for $a \in H^{0}(X, Y)$ that $\kappa(a)=r \circ \sigma(a)=\tilde{r}(a)$. As a consequence, if $X$ is a conjugation space, then $\pi_{0}\left(X^{\tau}\right) \approx \pi_{0}(X)$. This implies that $\tau$ preserves each path-connected component of $X$.

Remark 3.2 Let $X$ be an path-connected space with an involution $\tau$. Suppose that $X^{\tau}$ is non-empty and path-connected. Let $p t \in X^{\tau}$. Then, $X$ is a conjugation space if and only if $(X, p t)$ is a conjugation pair.

The remainder of this section is devoted to establishing the fundamental facts about conjugation pairs and spaces, and providing several important examples.

Theorem 3.3 (The multiplicativity theorem) Let $(\kappa, \sigma)$ be a $H^{*}$-frame for a conjugation $\tau$ on a pair $(X, Y)$. Then $\kappa$ and $\sigma$ are ring homomorphisms.

Proof We first prove that

$$
\sigma(a b)=\sigma(a) \sigma(b)
$$


for all $a \in H^{2 k}(X, Y)$ and $b \in H^{2 l}(X, Y)$. Let $m=k+l$. Since $\rho: H_{C}^{0}(X, Y) \rightarrow$ $H^{0}(X, Y)$ is an isomorphism, Equation (3.2) holds for $m=0$, and thus we may assume that $m>0$. As one has $\rho \circ \sigma(a b)=\rho(\sigma(a) \sigma(b))$, Equation (3.2) holds true modulo ker $\rho$ which is the ideal generated by $u$. As $H^{*}(X, Y)$ is concentrated in even degrees, this means that

$$
\sigma(a b)=\sigma(a) \sigma(b)+\sigma\left(d_{2 m-2}\right) u^{2}+\cdots+\sigma\left(d_{0}\right) u^{2 m},
$$

with $d_{i} \in H^{i}(X, Y)$. We must prove that $d_{2 m-2}=\cdots=d_{0}=0$.

Let us apply $r: H_{C}^{*}(X, Y) \rightarrow H_{C}^{*}\left(X^{\tau}, Y^{\tau}\right)$ to Equation (3.3). The left hand side gives

$$
r \circ \sigma(a b)=\kappa(a b) u^{m}+\ell t_{m}
$$

while the right hand side gives

$$
r \circ \sigma(a b)=\kappa(a) \kappa(b) u^{m}+\ell t_{m}+\left(\kappa\left(d_{2 m-2}\right) u^{m-1}+\ell t_{m-1}\right) u^{2}+\cdots+\kappa\left(d_{0}\right) u^{2 m} .
$$

Equations (3.4) and (3.5) imply that

$$
r \circ \sigma(a b)=\kappa\left(d_{0}\right) u^{2 m}+\ell t_{2 m} .
$$

Comparing Equations (3.4) and (3.6), we deduce that $d_{0}=0$, since $\kappa$ is injective. Then Equation (3.3) implies that

$$
r \circ \sigma(a b)=\kappa\left(d_{2}\right) u^{2 m-1}+\ell t_{2 m-1} .
$$

Again, comparing Equations (3.4) and (3.7), we deduce that $d_{2}=0$. This process continues until $d_{2 m-2}$, showing that each $d_{i}$ vanishes in Equation (3.3), which proves that $\sigma(a b)=\sigma(a) \sigma(b)$.

To establish that $\kappa(a b)=\kappa(a) \kappa(b)$ for $a, b$ as above, we use the fact that $r \circ \sigma(a b)=r \circ \sigma(a) \cdot r \circ \sigma(b)$ together with Equation (3.1) to conclude that

$$
\kappa(a b) u^{m}+\ell t_{m}=\left(\kappa(a) u^{k}+\ell t_{k}\right)\left(\kappa(b) u^{l}+\ell t_{l}\right)=\kappa(a) \kappa(b) u^{m}+\ell t_{m} .
$$

Therefore, $\kappa$ is multiplicative.

By the Leray-Hirsch theorem, the section $\sigma$ gives rise to an isomorphism of $R$-modules

$$
\hat{\sigma}: H^{*}(X, Y)[u] \stackrel{\approx}{\rightarrow} H_{C}^{*}(X, Y)
$$

(see (2.3) ). As $\sigma$ is a ring homomorphism by Theorem 3.3. one has the following corollary, which completely computes the ring $H_{C}^{*}(X, Y)$ in terms of $H^{*}(X, Y)$.

Corollary 3.4 Let $(\kappa, \sigma)$ be a $H^{*}$-frame for a conjugation on a pair $(X ; Y)$. Then $\hat{\sigma}: H^{*}(X, Y)[u] \stackrel{\approx}{\rightarrow} H_{C}^{*}(X, Y)$ is an isomorphism of $R$-algebras. 
Finally, there is a unique map $\hat{\kappa}: H_{C}^{2 *}(X, Y) \rightarrow H_{C}^{*}\left(X^{\tau}, Y^{\tau}\right)$ such that the following diagram

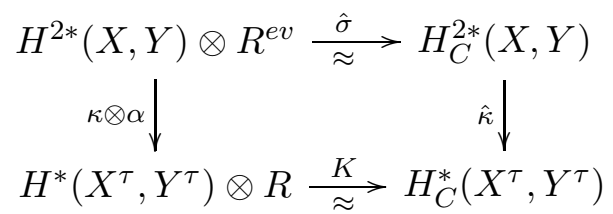

is commutative, where $K$ comes from the Künneth formula. The map $\hat{\kappa}$ is an isomorphism of $\left(R^{e v}, R\right)$-algebras over $\alpha: R^{e v} \rightarrow R$.

We now turn to examples of conjugation spaces and pairs.

Example 3.5 Conjugation cells Let $D=D^{2 k}$ be the closed disk of radius 1 in $\mathbb{R}^{2 k}$, equipped with an involution $\tau$ which is topologically conjugate to a linear involution with exactly $k$ eigenvalues equal to -1 . We call such a disk a conjugation cell of dimension $2 k$. Let $S$ be the boundary of $D$. The fixed point set is then homeomorphic to a disk of dimension $k$.

As $H^{*}(D, S)$ is concentrated in degree $2 k$, the restriction homomorphism $\rho: H_{C}^{2 k}(D, S) \rightarrow H^{2 k}(D, S)$ is an isomorphism. Set $\sigma=\rho^{-1}: H^{2 k}(D, S) \rightarrow$ $H_{C}^{2 k}(D, S)$. This shows that $(D, S)$ is equivariantly formal. The cohomology $H^{*}\left(D^{\tau}, S^{\tau}\right)$ is itself concentrated in degree $k$ and thus $H_{C}^{*}\left(D^{\tau}, S^{\tau}\right)=$ $H^{k}\left(D^{\tau}, S^{\tau}\right)[u]=\mathbb{Z}_{2}[u]$. The isomorphism $\kappa: H^{2 k}(D, S) \rightarrow H^{k}\left(D^{\tau}, S^{\tau}\right)$ is obvious. As $(D, S)$ is equivariantly formal, the restriction homomorphism $r: H_{C}^{*}(D, S) \rightarrow H_{C}^{*}\left(D^{\tau}, S^{\tau}\right)$ is injective. This is a consequence of the localization theorem for singular cohomology, which holds for smooth actions on compact manifolds. Therefore, if $a$ is the non-zero element of $H^{2 k}(D, S)$, the equation $r \sigma(a)=\kappa(a) u^{k}$ holds trivially. Hence, $(D, S)$ is a conjugation pair.

Example 3.6 Conjugation spheres If $D$ is a conjugation cell of dimension $2 k$ with boundary $S$, the quotient space $\Sigma=D / S$ is a conjugation space homeomorphic to the sphere $S^{2 k}$, while $\Sigma^{\tau}$ is homeomorphic to $S^{k}$. For $a \in$ $H^{2 k}(\Sigma)$, the conjugation equation $r \circ \sigma(a)=\kappa(a) u^{k}$ holds. We call such $\Sigma$ a conjugation sphere.

Example 3.7 Projective spaces Let us consider the complex projective space $\mathbb{C} P^{k}$ with the involution complex conjugation, having $\mathbb{R} P^{k}$ as real locus. One has $H^{2 *}\left(\mathbb{C} P^{k}\right)=\mathbb{Z}_{2}[a] /\left(a^{k+1}\right)$ and and $H^{*}\left(\mathbb{R} P^{k}\right)=\mathbb{Z}_{2}[b] /\left(b^{k+1}\right)$. The quotient 
space $\mathbb{C} P^{k} / \mathbb{C} P^{k-1}$ is a conjugation sphere. Hence, in the following commutative diagram,

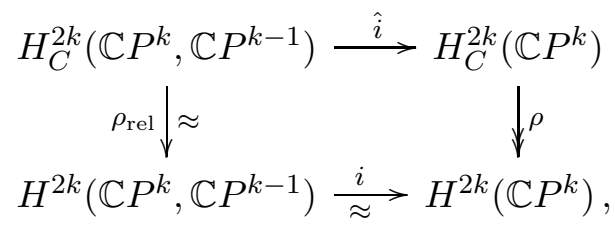

the map $\rho_{\text {rel }}$ is an isomorphism and hence $\rho$ is surjective. Setting $\sigma_{\text {rel }}:=\rho_{\text {rel }}^{-1}$, one gets a section $\sigma$ of $\rho$ by $\sigma:=\hat{i}_{\circ} \sigma_{\text {rel }} \circ i$. The isomorphism

$$
\kappa_{\text {rel }}: H^{2 *}\left(\mathbb{C} P^{k}, \mathbb{C} P^{k-1}\right) \stackrel{\approx}{\longrightarrow} H^{*}\left(\mathbb{R} P^{k}, \mathbb{R} P^{k-1}\right)
$$

is obvious and satisfies $i^{\tau} \circ \kappa_{\text {rel }}=\kappa \circ i$, where $\kappa: H^{2 *}\left(\mathbb{C} P^{k}\right) \stackrel{\approx}{\longrightarrow} H^{*}\left(\mathbb{R} P^{k}\right)$ is the unique ring isomorphism satisfying $\kappa(a)=b$. Moreover, using $\hat{\sigma}_{\text {rel }}$, we have $H_{C}^{*}\left(\mathbb{C} P^{k}, \mathbb{C} P^{k-1}\right)=H^{2 k}\left(\mathbb{C} P^{k}, \mathbb{C} P^{k-1}\right)[u]$, and using the Künneth formula, $H^{*}\left(\mathbb{R} P^{k}, \mathbb{R} P^{k-1}\right)=H^{k}\left(\mathbb{R} P^{k}, \mathbb{R} P^{k-1}\right)[u]$. Let $c \in H^{2 k}\left(\mathbb{C} P^{k}, \mathbb{C} P^{k-1}\right)$ and $c^{\prime} \in H^{k}\left(\mathbb{R} P^{k}, \mathbb{R} P^{k-1}\right)$ be the non-zero elements. As $\mathbb{C} P^{k} / \mathbb{C} P^{k-1}$ is a conjugation sphere, the equation $r \circ \sigma_{\text {rel }}(c)=c^{\prime} u^{k}$ holds, giving the formula $r \circ \sigma\left(a^{k}\right)=b^{k} u^{k}$ in $H_{C}^{2 k}\left(\mathbb{R} P^{k}\right)$.

Now, if $k \leq n \leq \infty$, the restriction homomorphisms $H^{2 *}\left(\mathbb{C} P^{n}\right) \rightarrow H^{2 *}\left(\mathbb{C} P^{k}\right)$, $H_{C}^{2 *}\left(\mathbb{C} P^{n}\right) \rightarrow H_{C}^{2 *}\left(\mathbb{C} P^{k}\right), H^{*}\left(\mathbb{R} P^{n}\right) \rightarrow H^{*}\left(\mathbb{R} P^{k}\right)$ and $H_{C}^{*}\left(\mathbb{R} P^{n}\right) \rightarrow H_{C}^{*}\left(\mathbb{R} P^{k}\right)$ are isomorphisms for $* \leq k$. Therefore, the equation $r \circ \sigma\left(a^{k}\right)=b^{k} u^{k}$ holds in $H_{C}^{2 k}\left(\mathbb{R} P^{n}\right)$ modulo elements in the kernel of the restriction homomorphism $H_{C}^{2 k}\left(\mathbb{R} P^{n}\right) \rightarrow H_{C}^{2 k}\left(\mathbb{R} P^{k}\right)$. This kernel consists of terms of type $\ell t_{k}$. Therefore, one has $r_{\circ} \sigma\left(a^{k}\right)=b^{k} u^{k}+\ell t_{k}=\kappa\left(a^{k}\right) u^{k}+\ell t_{k}$ which shows that $\mathbb{C} P^{n}$ is a conjugation space for all $n \leq \infty$.

We now show that the terms $\ell t_{k}$ in $H_{C}^{2 k}\left(\mathbb{R} P^{n}\right)$ never vanish when $n \geq 2 k$. Let $\rho^{\tau}: H_{C}^{*}\left(\mathbb{R} P^{n}\right) \rightarrow H^{*}\left(\mathbb{R} P^{n}\right)$ and $r_{0}: H^{*}\left(\mathbb{C} P^{n}\right) \rightarrow H^{*}\left(\mathbb{R} P^{n}\right)$ be the restriction homomorphisms. One has $\rho^{\tau} \circ r_{\circ} \sigma=r_{0} \circ \rho \circ \sigma$ and it is classical that $r_{0}(a)=b^{2}$ ( $a$ is the $(\bmod 2)$ Euler class of the Hopf bundle $\eta$ over $\mathbb{C} P^{\infty}$ and $b$ is the Euler class of the real Hopf bundle $\eta^{\tau}$ over $\mathbb{R} P^{\infty}$; these bundles satisfy $\eta_{\mathbb{R} P}=$ $\left.\eta^{\tau} \oplus \eta^{\tau}\right)$. Therefore, $r(a)=b u+b^{2}$. Since $r \circ \sigma$ is a ring homomorphism by Theorem 3.3. one has

$$
r \circ \sigma\left(a^{k}\right)=\left(b u+b^{2}\right)^{k} .
$$

Therefore, a term $b^{2 k}$ is always present in the right hand side of (3.10) when $n \geq 2 k$. For instance, $r \circ \sigma\left(a^{2}\right)=b^{2} u^{2}+b^{4}, r \circ \sigma\left(a^{3}\right)=b^{3} u^{3}+b^{4} u^{2}+b^{5} u+b^{6}$, and so on.

We finish this section with two related results. 
Lemma 3.8 (Injectivity lemma) Let $(X, Y)$ be a conjugation pair. Then the restriction homomorphism $r: H_{C}^{*}(X, Y) \rightarrow H_{C}^{*}\left(X^{\tau}, Y^{\tau}\right)$ is injective.

Proof Suppose that $r$ is not injective. Let $0 \neq x=\sigma(y) u^{k}+\ell t_{k} \in H_{C}^{2 n+k}(X, Y)$ be an element in $\operatorname{ker} r$. The conjugation equation guarantees that $k \neq 0$. We may assume that $k$ is minimal. By the conjugation equation again, we have $0=r(x)=\kappa(y) u^{n+k}+\ell t_{n+k}$. Since $\kappa$ is an isomorphism, we get $y=0$, which is a contradiction.

Lemma 3.9 Let $(X, Y)$ be a conjugation pair. Assume that $H^{2 *}(X, Y)=0$ for $*>m_{0}$. Then the localization theorem holds. That is, the restriction homomorphism $r: H_{C}^{*}(X, Y) \rightarrow H_{C}^{*}\left(X^{\tau}, Y^{\tau}\right)$ becomes an isomorphism after inverting $u$.

Proof By Lemma 3.8 it suffices to show that

$$
H^{*}\left(X^{\tau}, Y^{\tau}\right)=H^{*}\left(X^{\tau}, Y^{\tau}\right) \otimes 1 \subset H_{C}^{*}\left(X^{\tau}, Y^{\tau}\right)
$$

is in the image of $r$ localized. We show this by downward induction on the degree of an element in $H^{*}\left(X^{\tau}, Y^{\tau}\right)$. The statement is obvious for $*>m_{0}$. Since $r_{\circ} \sigma(x)=\kappa(x) u^{k}+\ell t_{k}$ for $x \in H^{2 k}(X, Y)$, the induction step follows (by induction hypothesis, $\ell t_{k}$ is in the image of $r$ localized).

Remark 3.10 In classical equivariant cohomology theory, the injectivity lemma is often deduced from the localization theorem. But, as seen in Example 3.7 $\mathbb{C} P^{\infty}$ with the complex conjugation is a conjugation space, and therefore satisfies the injectivity lemma. However, $r_{\text {loc }}: H_{C}^{*}\left(\mathbb{C} P^{\infty}\right)\left[u^{-1}\right] \rightarrow H_{C}^{*}\left(\mathbb{R} P^{\infty}\right)\left[u^{-1}\right]$ is not surjective. Indeed, $H_{C}^{*}\left(\mathbb{C} P^{\infty}\right)\left[u^{-1}\right]=\mathbb{Z}_{2}\left[a, u, u^{-1}\right], H_{C}^{*}\left(\mathbb{R} P^{\infty}\right)\left[u^{-1}\right]=$ $\mathbb{Z}_{2}\left[b, u, u^{-1}\right]$ and $r_{\text {loc }}(a)=b u+b^{2}$ by Example 3.7. Therefore, $r_{\text {loc }}$ composed with the epimorphism $\mathbb{Z}_{2}\left[b, u, u^{-1}\right] \rightarrow \mathbb{Z}_{2}$ sending $b$ and $u$ to 1 is the zero map.

\subsection{Equivariant maps between conjugation spaces}

The purpose of this section is to show the naturality of $H^{*}$-frames. Let $\left(X, X_{0}\right)$ and $\left(Y, Y_{0}\right)$ be two conjugation pairs. Choose $H^{*}$-frames $\left(\kappa_{X}, \sigma_{X}\right)$ and $\left(\kappa_{Y}, \sigma_{Y}\right)$ for $\left(X, X_{0}\right)$ and $\left(Y, Y_{0}\right)$ respectively. Let $f:\left(Y, Y_{0}\right) \rightarrow\left(X, X_{0}\right)$ be a $C$ equivariant map of pairs. We denote by $f^{\tau}:\left(Y^{\tau}, Y_{0}^{\tau}\right) \rightarrow\left(X^{\tau}, X_{0}^{\tau}\right)$ the restriction of $f$ to $\left(Y^{\tau}, Y_{0}^{\tau}\right)$ and use the functorial notations : $H^{*} f, H_{C}^{*} f$, and so forth. 
Proposition 3.11 The conjugation space structure of a conjugation space is natural, i.e., one has

$$
H_{C}^{*} f \circ \sigma_{X}=\sigma_{Y} \circ H^{*} f
$$

and

$$
H^{*} f^{\tau} \circ \kappa_{X}=\kappa_{Y} \circ H^{*} f
$$

Proof Let $\rho_{X}: H_{C}^{*}\left(X, X_{0}\right) \rightarrow H^{*}\left(X, X_{0}\right)$ and $\rho_{Y}: H_{C}^{*}\left(Y, Y_{0}\right) \rightarrow H^{*}\left(Y, Y_{0}\right)$ denote the restriction homomorphisms. Let $a \in H^{2 k}\left(X, X_{0}\right)$. As $H^{*} f \circ \rho_{X}=$ $\rho_{Y} \circ H_{C}^{*} f$, one has

$$
\rho_{Y} \circ H_{C}^{*} f \circ \sigma_{X}(a)=H^{*} f \circ \rho_{X} \circ \sigma_{X}(a)=H^{*} f(a)=\rho_{Y} \circ \sigma_{Y} \circ H^{*} f(a) .
$$

This implies that Equation (3.11) holds modulo the ideal $(u)$. As $H^{*}\left(X, X_{0}\right)$ is concentrated in even degrees, this means that

$$
H_{C}^{*} f \circ \sigma_{X}(a)=\sigma_{Y} \circ H^{*} f(a)+\sigma_{Y}\left(d_{2 k-2}\right) u^{2}+\cdots+\sigma_{Y}\left(d_{0}\right) u^{2 k},
$$

where $d_{i} \in H^{i}\left(Y, Y_{0}\right)$. Now, by Lemma 2.2 ,

$$
H_{C}^{*} f^{\tau} \circ r_{X} \circ \sigma_{X}(a)=H_{C}^{*} f^{\tau}\left(\kappa_{X}(a) u^{k}+\ell t_{k}\right)=H^{*} f^{\tau} \circ \kappa_{X}(a) u^{k}+\ell t_{k} .
$$

On the other hand, by equation (3.14)

$$
r_{Y} \circ H_{C}^{*} f(a)=\sigma_{Y}\left(d_{0}\right) u^{2 k}+\ell t_{2 k} .
$$

But $r_{Y} \circ H_{C}^{*} f=H_{C}^{*} f^{\tau} \circ r_{X}$. Comparing then Equation (3.16) with Equation (3.15), we deduce that $d_{0}=0$, since $\kappa_{Y}$ is an injective. Then

$$
r_{Y} \circ H_{C}^{*} f(a)=\kappa_{Y}\left(d_{2}\right) u^{2 k-2}+\ell t_{2 k-2} .
$$

Again, we deduce that $d_{2}=0$. Continuing this process, we finally get Equation (3.11) (as in the proof of Theorem (3.3)).

As for Equation (3.12), by Lemma 2.2 .

$$
H_{C}^{*} f^{\tau} \circ r_{X} \circ \sigma_{X}(a)=H_{C}^{*} f^{\tau}\left(\kappa_{X}(a) u^{k}+\ell t_{k}\right)=H^{*} f^{\tau} \circ \kappa_{X}(a) u^{k}+\ell t_{k} .
$$

On the other hand, using Equation (3.11),

$$
r_{Y} \circ H_{C}^{*} f \circ \sigma_{X}(a)=r_{Y} \circ \sigma_{Y} \circ H^{*} f(a)=\kappa_{Y} \circ H^{*} f(a) u^{k}+\ell t_{k} .
$$

Comparing Equation (3.18) with (3.19) gives Equation (3.12).

As a corollary of Proposition [3.11] we get the uniqueness of the conjugation space structure for a conjugation space.

Corollary 3.12 Let $(\kappa, \sigma)$ and $\left(\kappa^{\prime}, \sigma^{\prime}\right)$ be two $H^{*}$-frames for an involution $\tau$ on $\left(X, X_{0}\right)$ Then $(\kappa, \sigma)=\left(\kappa^{\prime}, \sigma^{\prime}\right)$ 
Proof If two $H^{*}$-frames $\left(\kappa_{X}, \sigma_{X}\right)$ and $\left(\kappa_{X}^{\prime}, \sigma_{X}^{\prime}\right)$ are given on $\left(X, X_{0}\right)$, Proposition 3.11 with $f=\mathrm{id}_{X}$ proves that $\kappa_{X}=\kappa_{X}^{\prime}$ and $\sigma_{X}=\sigma_{X}^{\prime}$.

By the Leray-Hirsch Theorem, the section $\sigma_{X}: H^{*}\left(X, X_{0}\right) \rightarrow H_{C}^{*}\left(X, X_{0}\right)$ induces a map $\hat{\sigma}_{X}: H^{*}\left(X, X_{0}\right)[u] \stackrel{\approx}{\rightarrow} H_{C}^{*}\left(X, X_{0}\right)$ which is an isomorphism of $R$-algebras by Corollary 3.4. We define $\hat{\sigma}_{Y}: H^{*}\left(Y, Y_{0}\right)[u] \stackrel{\approx}{\rightarrow} H_{C}^{*}\left(Y, Y_{0}\right)$ accordingly. Proposition 3.11 shows that these $R$-algebras isomorphisms are natural and gives the following analogue of Lemma 2.2

Corollary 3.13 For any $C$-equivariant map $f: Y \rightarrow X$ between conjugation spaces, the diagram

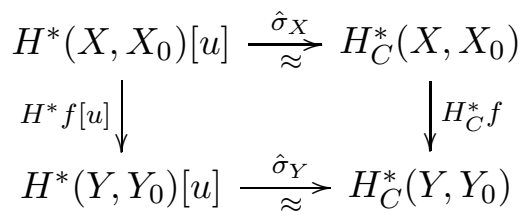

is commutative, where $H^{*} f[u]$ is the polynomial extension of $H^{*} f$.

Finally, Proposition 3.11 and Corollary 3.13 give the naturality of the algebra isomorphism $\hat{\kappa}$ of Equation (3.8).

Proposition 3.14 For any $C$-equivariant map $f: Y \rightarrow X$ between conjugation spaces, the diagram

$$
\begin{array}{ccc}
H_{C}^{2 *}\left(X, X_{0}\right) & \stackrel{H_{C}^{2 *} f}{\longrightarrow} & H_{C}^{2 *}\left(Y, Y_{0}\right) \\
\downarrow \hat{\kappa}_{X} & & \downarrow \hat{\kappa}_{Y} \\
H_{C}^{*}\left(X^{\tau}, X_{0}^{\tau}\right) & \stackrel{H_{C}^{*} f^{\tau}}{\longrightarrow} & H_{C}^{*}\left(Y^{\tau}, Y_{0}^{\tau}\right)
\end{array}
$$

is commutative.

\section{Extension properties}

\subsection{Triples}

Proposition 4.1 Let $X$ be a space with an involution $\tau$ and let $Z \subset Y$ be $\tau$-invariant subspaces of $X$. Suppose that $(X, Y)$ and $(Y, Z)$ are conjugation pairs. Then $(X, Z)$ is a conjugation pair. 
Proof The subscript " $X, Y$ " is used for the relevant homomorphism for the pair $(X, Y)$, like $\kappa_{X, Y}, r_{X, Y}$, etc. In order to simplify the notation, we use the subscripts " $X$ " or " $Y$ " for the pairs $(X, Z)$ and $(Y, Z)$, as if $Z$ were empty. Thus, we must construct a $H^{*}$-frame $\left(\kappa_{X}, \sigma_{X}\right)$ for the pair $(X, Z)$, using those $\left(\kappa_{Y}, \sigma_{Y}\right)$ and $\left(\kappa_{X, Y}, \sigma_{X, Y}\right)$ for the conjugation pairs $(Y, Z)$ and $(X, Y)$.

We first prove that the restriction homomorphisms $\hat{j}: H_{C}^{*}(X, Z) \rightarrow H_{C}^{*}(Y, Z)$ and $j^{\tau}: H^{*}\left(X^{\tau}, Z^{\tau}\right) \rightarrow H^{*}\left(Y^{\tau}, Z^{\tau}\right)$ are surjective. Let us consider the following commutative diagram

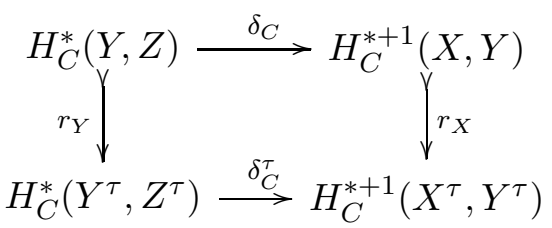

in which $\delta_{C}$ and $\delta_{C}^{\tau}$ are the connecting homomorphisms for the long exact sequences in equivariant cohomology of the triples $(X, Y, Z)$ and $\left(X^{\tau}, Y^{\tau}, Z^{\tau}\right)$ respectively. The vertical restriction homomorphisms are injective by Lemma 3.8 Clearly, $\delta_{C}=0$ if and only if $\hat{j}$ is surjective. As $\delta_{C}^{\tau}$ is the polynomial extension of $\delta^{\tau}$, one also has

$$
\delta_{C}^{\tau}=0 \Longleftrightarrow \delta^{\tau}=0 \Longleftrightarrow j^{\tau} \text { is surjective. }
$$

As $(X, Y)$ is an even cohomology pair, for $y \in H^{2 k}(Y, Z)$, one can write

$$
\delta_{C} \circ \sigma_{Y}(y)=\sum_{i=0}^{k} \sigma_{X}\left(x_{2 k-2 i}\right) u^{2 i+1},
$$

with $x_{2 k-2 i} \in H^{2 k-2 i}(X, Y)$. Using that $r_{Y} \circ \sigma_{Y}(y)=\kappa_{Y}(y) u^{k}+\ell t_{k}$, the commutativity of Diagram (4.1) and that $\delta_{C}^{\tau}=\delta^{\tau}[u]$, we get

$$
\begin{aligned}
\delta^{\tau} \circ \kappa_{Y}(y) u^{k}+\ell t_{k} & =\delta_{C}^{\tau}\left(\kappa_{Y}(y) u^{k}+\ell t_{k}\right) \\
& =r_{X}\left(\sum_{i=0}^{k} \sigma_{X}\left(x_{2 k-2 i}\right) u^{2 i+1}\right) \\
& =\sum_{i=0}^{k}\left(\kappa_{X}\left(x_{2 k-2 i}\right) u^{k+i+1}+\ell t_{k+i+1}\right) .
\end{aligned}
$$

As in the proof of Theorem 3.3. we compare the coefficients of powers of $u$, in both sides of Equation 4.3, Starting with $u^{2 k+1}$ and going downwards, we get inductively that $\kappa_{X}\left(x_{2 k-2 i}\right)=0$ for $i=0, \ldots, k$. Hence $x_{2 k-2 i}=0$ for $i=0, \ldots, k$ and the right side of Equation 4.3 vanishes for all $y \in H^{2 k}(Y, Z)$. As $\kappa_{Y}$ is bijective, we deduce that $\delta^{\tau}=0$ and $\delta_{C}^{\tau}=0$. As $r_{X}$ is injective by 
Lemma 3.8, the commutativity of Diagram (4.1) implies that $\delta_{C}=0$. We have thus proven that the restriction homomorphisms $\hat{j}: H_{C}^{*}(X, Z) \rightarrow H_{C}^{*}(Y, Z)$ and $j^{\tau}: H^{*}\left(X^{\tau}, Z^{\tau}\right) \rightarrow H^{*}\left(Y^{\tau}, Z^{\tau}\right)$ are surjective.

As $\rho_{Y}$ is onto, the cohomology exact sequence of $(X, Y, Z)$ decomposes into short exact sequences and one has the following commutative diagram:

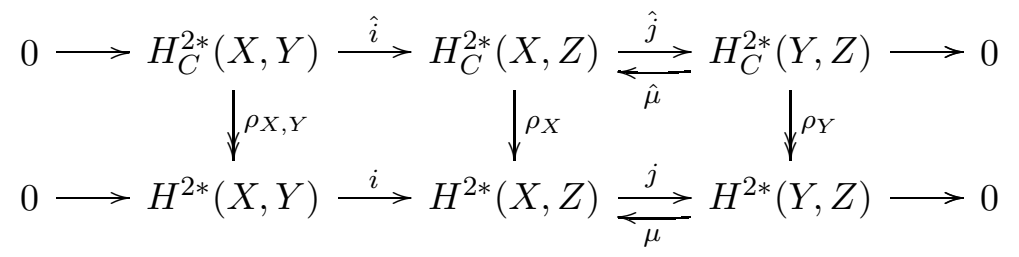

Sections $\hat{\mu}$ and $\mu$ can be constructed as follows. Let $\mathcal{B}$ be a basis of the $\mathbb{Z}_{2}$ vector space $H^{2 *}(Y, Z)$. The set $\sigma_{Y}(B)$ is a $R^{e v}$-module basis for $H_{C}^{2 *}(Y, Z)$. For each $b \in \mathcal{B}$, choose $\tilde{b} \in H_{C}^{2 *}(X, Z)$ such that $\hat{j}(\tilde{b})=\sigma_{Y}(b)$. The correspondence $\sigma_{Y}(b) \rightarrow \tilde{b}$ induces a section $\hat{\mu}: H_{C}^{2 *}(Y, Z) \rightarrow H_{C}^{2 *}(X, Z)$ of $\hat{j}$. One has $j \circ \rho_{X} \circ \hat{\mu} \circ \sigma_{Y}(b)=b$; therefore $\mu:=\rho_{X} \circ \hat{\mu} \circ \sigma_{Y}$ is an additive section of the epimorphism $j$.

Using that additively, $H^{2 *}(X, Z)=i\left(H^{2 *}(X, Y)\right) \oplus \mu\left(H^{2 *}(Y, Z)\right)$, one defines $\sigma_{X}^{0}: H^{2 *}(X, Z) \rightarrow H_{C}^{2 *}(X, Z)$ by:

$$
\left\{\begin{aligned}
\sigma_{X}^{0}(i(a)) & :=\hat{i}_{\circ} \sigma_{X, Y}(a) & & \text { for all } a \in H^{2 *}(X, Y) \\
\sigma_{X}^{0}(\mu(b)) & :=\hat{\mu} \circ \sigma_{Y}(b) & & \text { for all } b \in H^{2 *}(Y, Z)
\end{aligned}\right.
$$

The map $\sigma_{X}^{0}$ is an additive section of $\rho_{X}$ and the following diagram is commutative:

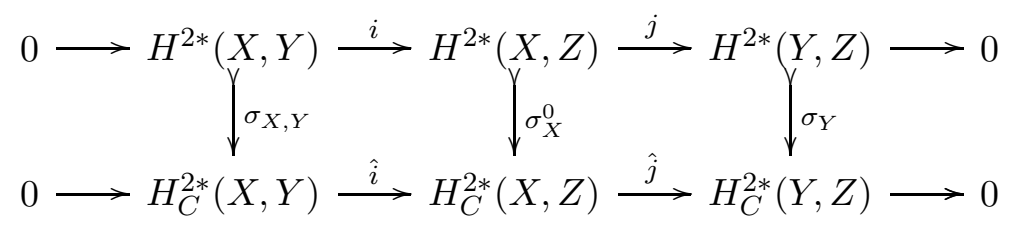

We define an additive map $\kappa_{X}^{0}: H^{2 *}(X, Z) \rightarrow H^{*}\left(X^{\tau}, Z^{\tau}\right)$ by

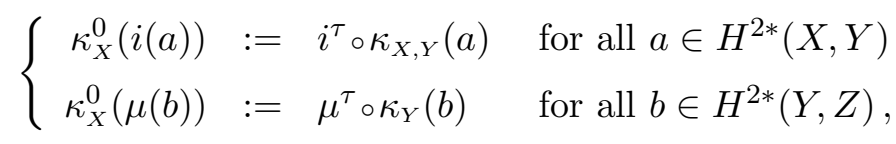

where $\mu^{\tau}: H^{*}\left(Y^{\tau}, Z^{\tau}\right) \rightarrow H^{*}\left(X^{\tau}, Z^{\tau}\right)$ is any additive section of $j^{\tau}$. The fol- 
lowing diagram is then commutative:

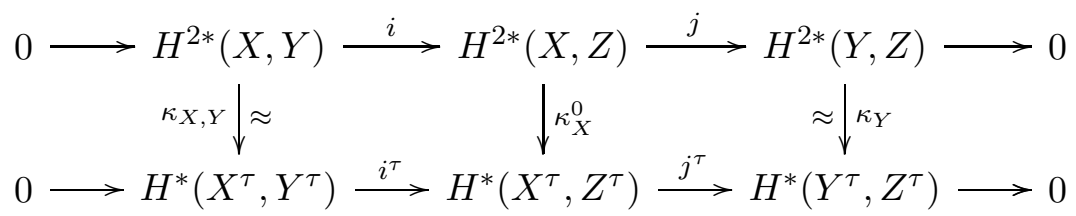

By construction, the equality $r_{X} \circ \sigma_{X}^{0}(i(a))=\kappa_{X}^{0}(i(a)) u^{k}+\ell t_{k}$ holds for all $a \in H^{2 k}(X, Y)$ and all $k$. On the other hand, for $b \in H^{2 k}(Y, Z)$, we only have that $j^{\tau} \circ r_{X} \circ \sigma_{X}^{0}(\mu(b))=j^{\tau}\left(\kappa_{X}^{0}(\mu(b)) u^{k}+\ell t_{k}\right)$, which implies that

$$
r_{X} \circ \sigma_{X}^{0}(\mu(b))=\hat{i}^{\tau}(D)+\kappa_{X}^{0}(\mu(b)) u^{k}+\ell t_{k}
$$

for some $D \in H_{C}^{*}\left(Y^{\tau}, Z^{\tau}\right)$. As $\hat{i}^{\tau}$ is injective (since $\delta_{C}^{\tau}$ is onto), the element $D$ in Equation (4.9) is unique if chosen free of terms $\ell t_{k}$. Such a $D$ is of the form

$$
D=\kappa_{X, Y}\left(d_{2 k}\right) u^{k}+\sum_{s=1}^{k} \kappa_{X, Y}\left(d_{2(k-s)}\right) u^{k+s},
$$

where $d_{i} \in H^{i}(X, Y)$. Define $\sigma_{X}: H^{2 *}(X, Z) \rightarrow H_{C}^{2 *}(X, Z)$ and $\kappa_{X}: H^{2 *}(X, Z)$ $\rightarrow H^{*}\left(X^{\tau}, Z^{\tau}\right)$ by

$$
\sigma_{X}(i(a)):=\sigma_{X}^{0}(i(a)) \text { and } \kappa_{X}(i(a))=\kappa_{X}^{0}(i(a)) \text { for all } a \in H^{2 *}(X, Y),
$$

and, for $b \in H^{2 *}(Y, Z)$, by

$$
\left\{\begin{array}{rl}
\sigma_{X}(\mu(b)) & :=\sigma_{X}^{0}(\mu(b))+\sum_{s=1}^{k} i\left(d_{2(k-s)}\right) u^{2 s} \\
\kappa_{X}(\mu(b)) & :=\kappa_{X}^{0}(\mu(b))+i^{\tau}\left(d_{2 k}\right)
\end{array} .\right.
$$

We may check that $r_{X} \circ \sigma_{X}(c)=\kappa_{X}(c) u^{k}+\ell t_{k}$ for all $c \in H^{2 k}(X, Z)$. As $\sigma_{X}(c)-\sigma_{X}^{0}(c) \in H^{*}(X, Z) \cdot u=\operatorname{ker} \rho_{X}$, the homomorphism $\sigma_{X}$ is a section of $\rho_{X}$. Diagram (4.8) still commutes with $\kappa_{X}$ instead of $\kappa_{X}^{0}$. As $\kappa_{X, Y}$ and $\kappa_{Y}$ are bijective, $\kappa_{X}$ is bijective by the five-lemma.

Proposition 4.2 Let $X$ be a space with an involution $\tau$ and let $Z \subset Y$ be $\tau$-invariant subsets of $X$. Suppose that

(i) $(X, Z)$ and $(X, Y)$ are conjugation pairs.

(ii) the restriction homomorphisms $i: H^{*}(X, Y) \rightarrow H^{*}(X, Z)$ is injective.

Then $(Y, Z)$ is a conjugation pair.

Remark 4.3 Assuming condition (i), condition (ii) is necessary for $(Y, Z)$ to be a conjugation pair, since the three pairs will then have cohomology only in even degrees. 
Proof of Proposition 4.2 We have the following commutative diagram

$$
\begin{array}{rrrr}
H_{C}^{2 *}(X, Y) \stackrel{\hat{i}}{\longrightarrow} H_{C}^{2 *}(X, Z) \stackrel{\hat{j}}{\longrightarrow} H_{C}^{2 *}(Y, Z) \\
\sigma_{X, Y} \uparrow \mid \rho_{X, Y} & \sigma_{X} \uparrow \downarrow \rho_{X} & \\
0 \longrightarrow & H^{2 *}(X, Y) \stackrel{i}{\longrightarrow} H^{2 *}(X, Z) \stackrel{j}{\rightleftarrows} H^{2 *}(Y, Z) \longrightarrow 0
\end{array}
$$

where $\mu$ is an additive section of $j$. Define an additive section $\sigma_{Y}$ of $\rho_{Y}$ by $\sigma_{Y}:=\hat{j} \circ \sigma_{X} \circ \mu$ and $\kappa_{Y}: H^{2 *}(Y, Z) \rightarrow H^{*}\left(Y^{\tau}, Z^{\tau}\right)$ by $\kappa_{Y}:=\hat{j}^{\tau} \circ \kappa_{X} \circ \mu$. This guarantees that $r_{Y} \sigma_{Y}(a)=\kappa_{Y}(a) u^{k}+\ell t_{k}$ for all $a \in H^{2 k}(Y, Z)$. It then just remains to prove that $\kappa_{Y}$ is bijective.

As $i$ is injective, the equation $i^{\tau} \circ \kappa_{X, Y}=\kappa_{X} \circ i$, guaranteed by Proposition 3.11 implies that $i^{\tau}$ is injective. The same equation implies that $j^{\tau} \circ \kappa_{X}=\kappa_{Y} \circ j$, since $j^{\tau} \circ \kappa_{X} \circ i=0$. Therefore, one has a commutative diagram

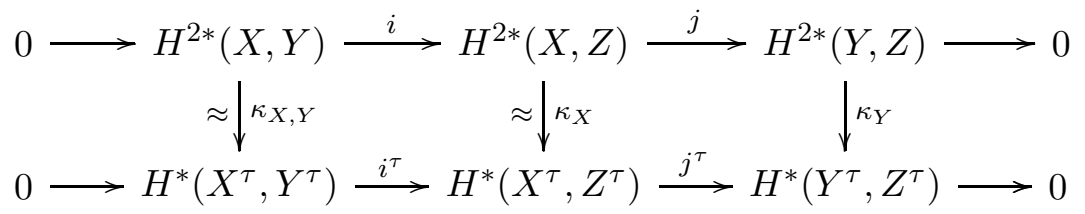

which shows that $\kappa_{Y}$ is bijective.

The same kind of argument will prove Proposition 4.4 below. As this proposition is not used elsewhere in this paper, we leave the proof to the reader.

Proposition 4.4 Let $X$ be a space with an involution $\tau$ and let $Z \subset Y$ be $\tau$-invariant subsets of $X$. Suppose that

(i) $(X, Z)$ and $(Y, Z)$ are conjugation pairs.

(ii) the restriction homomorphisms $j: H^{*}(X, Z) \rightarrow H^{*}(Y, Z)$ is surjective.

Then $(X, Y)$ is a conjugation pair.

\subsection{Products}

Proposition 4.5 Let $\left(X, X_{0}\right)$ and $\left(Y, Y_{0}\right)$ be conjugation pairs. Suppose that $H^{q}\left(X, X_{0}\right)$ is finite dimensional for each $q$. Assume that $\left\{X \times Y_{0}, X_{0} \times Y\right\}$ is an excisive couple in $X \times Y$ and that $\left\{X^{\tau} \times Y_{0}^{\tau}, X_{0}^{\tau} \times Y^{\tau}\right\}$ is an excisive couple in $X^{\tau} \times Y^{\tau}$. Then, the product pair $\left(X \times Y,\left(X_{0} \times Y\right) \cup\left(X \times Y_{0}\right)\right)$ is a conjugation pair. 
Proof To simplify the notations, we give the proof when $X_{0}=Y_{0}=\emptyset$; the general case is identical. By of our hypotheses, the two projections $X \times Y \rightarrow X$ and $X \times Y \rightarrow Y$ give rise to the Künneth isomorphism

$$
K: H^{*}(X) \otimes H^{*}(Y) \stackrel{\approx}{\longrightarrow} H^{*}(X \times Y) .
$$

The same holds for the fixed point sets, producing

$$
K^{\tau}: H^{*}\left(X^{\tau}\right) \otimes H^{*}\left(Y^{\tau}\right) \stackrel{\approx}{\longrightarrow} H^{*}\left(X^{\tau} \times Y^{\tau}\right)=H^{*}\left((X \times Y)^{\tau}\right) .
$$

The Borel construction applied to the projections gives rise to maps $(X \times Y)_{C} \rightarrow$ $X_{C}$ and $(X \times Y)_{C} \rightarrow Y_{C}$. This produces a ring homomorphism

$$
K_{C}: H^{*}\left(X_{C}\right) \otimes H^{*}\left(Y_{C}\right) \longrightarrow H^{*}\left((X \times Y)_{C}\right) .
$$

We now want to define $\kappa_{X \times Y}$ and $\sigma_{X \times Y}$. We set $\kappa_{X \times Y}:=K^{\tau} \circ\left(\kappa_{X} \otimes \kappa_{Y}\right) \circ K^{-1}$. Then, $\kappa_{X \times Y}$ is an isomorphism and one has the following commutative diagram:

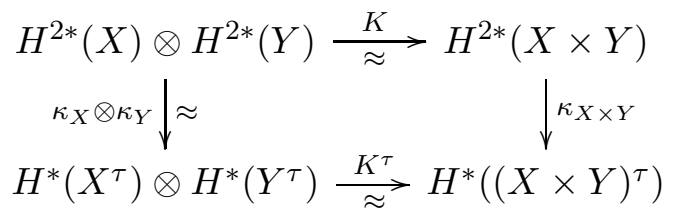

Now, setting $\sigma_{X \times Y}:=K_{C} \circ\left(\sigma_{X} \otimes \sigma_{Y}\right) \circ K^{-1}$, we have:

$$
\begin{array}{rr}
H_{C}^{2 *}(X) \otimes H_{C}^{2 *}(Y) \stackrel{K_{C}}{\longrightarrow} H_{C}^{2 *}(X \times Y) \\
\rho_{X} \otimes \rho_{Y} \downarrow \uparrow \sigma_{X} \otimes \sigma_{Y} & \rho_{X \times Y} \downarrow \uparrow \sigma_{X \times Y} \\
H^{2 *}(X) \otimes H^{2 *}(Y) \stackrel{K}{\underset{\sim}{\longrightarrow}} H^{2 *}(X \times X)
\end{array}
$$

With these definitions, one verifies the conjugation equation by direct computation.

\subsection{Direct limits}

Proposition 4.6 Let $\left(X_{i}, f_{i j}\right)$ be a directed system of conjugation spaces and $\tau$-equivariant inclusions, indexed by a direct set $\mathcal{I}$. Suppose that each space $X_{i}$ is $T_{1}$. Then $X=\lim X_{i}$ is a conjugation space.

Proof As the maps $f_{i j}$ are inclusion between and each $X_{i}$ is $T_{1}$, the image of a compact set $K$ under a continuous map to $X$ is contained in some $X_{i}$ 
(otherwise $K$ would contain an infinite closed discrete subspace). Therefore, $H_{*}(X)=\lim _{\rightarrow} H_{*}\left(X_{i}\right)$ (singular homology with $\mathbb{Z}_{2}$ as coefficients). Then

$$
\begin{aligned}
H^{*}(X)=\operatorname{Hom}\left(H_{*}(X) ; \mathbb{Z}_{2}\right) & =\operatorname{Hom}\left(\lim _{\rightarrow} H_{*}\left(X_{i}\right) ; \mathbb{Z}_{2}\right) \\
& =\lim \operatorname{Hom}\left(H_{*}\left(X_{i}\right) ; \mathbb{Z}_{2}\right) \\
& =\lim _{\leftarrow} H^{*}\left(X_{i}\right) .
\end{aligned}
$$

One has $X^{\tau}=\lim _{\rightarrow} X_{i}^{\tau}$ and $X_{C}=\lim _{\rightarrow}\left(X_{i}\right)_{C}$ and, as in (4.13), one has $H^{*}\left(X^{\tau}\right)=\lim _{\leftarrow} H^{*}\left(X_{i}^{\tau}\right)$ and $H_{C}^{*}(X)=\lim _{\leftarrow} H_{C}^{*}\left(X_{i}\right)$. By Proposition 3.11] the isomorphisms $\kappa_{i}: H^{2 *}\left(X_{i}\right) \rightarrow H^{*}\left(X_{i}^{\tau}\right)$ is an isomorphism of inverse systems; we can thus define $\kappa=\lim _{\leftarrow} \kappa_{i}$, and $\kappa$ is an isomorphism. The same can be done for $\sigma: H^{2 *}(X) \rightarrow H_{C}^{2 *}(X)$, defined, using Proposition 3.11] as the inverse limit of $\sigma_{i}: H^{2 *}\left(X_{i}\right) \rightarrow H_{C}^{2 *}\left(X_{i}\right)$, and $\sigma$ is a section of $\rho: H_{C}^{2 *}(X) \rightarrow$ $H^{2 *}(X)$. The conjugation equation for $(\sigma, \kappa)$ comes directly from that for $\left(\sigma_{i}, \kappa_{i}\right)$.

\subsection{Equivariant connected sums}

Let $M$ be a smooth oriented closed manifold of dimension $2 k$ together with a smooth involution $\tau$ that is a conjugation. Then $M^{\tau}$ is a non-empty closed submanifold of $M$ of dimension $k$. Pick a point $p \in M^{\tau}$. There is a $\tau$ invariant disk $\Delta$ of dimension $2 k$ in $M$ around $p$ on which $\tau$ is conjugate to a linear action: there is a diffeomorphism $h: \mathbb{D}\left(\mathbb{R}^{k} \times \mathbb{R}^{k}\right) \rightarrow \Delta$ preserving the orientation such that $\tau \circ h=h \circ \tau_{0}$, where $\tau_{0}(x, y)=(x,-y)$.

Let $\left(M_{i}, \tau_{i}\right), i=1,2$, be two smooth conjugation spaces, as above. Choosing conjugation cells (see Example 3.5) $h_{i}: \mathbb{D}\left(\mathbb{R}^{k} \times \mathbb{R}^{k}\right) \rightarrow \Delta_{i}$ as above, one can form the connected sum

$$
M:=M_{1} \sharp M_{2}=\left(M_{1} \backslash \operatorname{int} \Delta_{1}\right) \cup_{h_{2} \circ h_{1}^{-1}}\left(M_{2} \backslash \operatorname{int} \Delta_{2}\right)
$$

which inherits an involution $\tau$. We do not know whether the equivariant diffeomorphism type of $M_{1} \sharp M_{2}$ depends on the choice of the diffeomorphism $h_{i}$, which is unique only up to pre-composition by elements of $S(O(k) \times O(k))$.

Proposition 4.7 $\quad M_{1} \sharp M_{2}$ is a conjugation space.

Proof Let $M=M_{1} \sharp M_{2}$ and let $N_{i}=M_{i} \backslash \operatorname{int} \Delta_{i}$. By excision, one has ring isomorphisms

$$
H^{*}\left(M, N_{1}\right) \stackrel{\approx}{\rightarrow} H^{*}\left(N_{2}, \partial N_{2}\right) \stackrel{\approx}{\leftarrow} H^{*}\left(M_{2}, \Delta_{2}\right) \stackrel{\approx}{\rightarrow} H^{*}\left(M_{2}, p_{2}\right) .
$$


The same isomorphisms hold for the $C$-equivariant cohomology and for the cohomology of the fixed point sets. As $M_{2}$ is a conjugation space, the pair $\left(M_{2}, p_{2}\right)$ is a conjugation pair by Remark 3.2. Therefore, $\left(M, N_{1}\right)$ is a conjugation pair.

Proposition 4.2 applied to $X=M_{1}, Y=N_{1}$ and $Z=\emptyset$ shows that $N_{1}$ is a conjugation space. Applying then Proposition 4.1 to $X=M, Y=N_{1}$ and $Z=\emptyset$ proves that $M$ is a conjugation space.

\section{Conjugation complexes}

\subsection{Attaching conjugation cells}

Let $D^{2 k}$ be the closed disk of radius 1 in $\mathbb{R}^{2 k}$, equipped with an involution $\tau$ which is topologically conjugate to a linear involution with exactly $k$ eigenvalues equal to -1. As seen in Example 3.5 we call such a disk a conjugation cell of dimension $2 k$. The fixed point set is then homeomorphic to a disk of dimension $k$. Observe that a product of two conjugation cells is a conjugation cell.

Let $Y$ be a topological space with an involution $\tau$. Let $\alpha: S^{2 k-1} \rightarrow Y$ be an equivariant map. Then the involutions on $Y$ and on $D^{2 k}$ induce an involution on the quotient space

$$
X=Y \cup_{\alpha} D^{2 k}=Y \amalg D^{2 k} /\left\{u=\alpha(u) \mid x \in S^{2 k-1}\right\} .
$$

We say that $X$ is obtained from $Y$ by attaching a conjugation cell of dimension $2 k$. Note that the real locus $X^{\tau}$ is obtained from $Y^{\tau}$ by adjunction of a $k$-cell. Attaching a conjugation cell of dimension 0 is making the disjoint union with a point.

More generally, one can attach to $Y$ a set $\Lambda$ of $2 k$-conjugation cells, via an equivariant map $\alpha: \amalg_{\Lambda} S_{\lambda}^{2 k-1} \rightarrow Y$. The resulting space $X$ is equipped with an involution and its real locus $X^{\tau}$ is obtained from $Y^{\tau}$ by adjunction of a collection of $k$-cells labeled by the same set $\Lambda$.

The main result of this section is the following:

Proposition 5.1 Let $(Y, Z)$ be a conjugation pair and let $X$ be obtained from $Y$ by attaching a collection of conjugation cells of dimension $2 k$. Then $(X, Z)$ is a conjugation pair. 
Proof Without loss of generality, we may assume that $Y$ and $X$ are pathconnected. We may also suppose that $Z$ and $Z^{\tau}$ are not empty. Indeed, if $Z \neq \emptyset$ then $Z^{\tau} \neq \emptyset$ since $H^{0}(Y, Z) \approx H^{0}\left(Y^{\tau}, Z^{\tau}\right)$. If $Z=\emptyset$, we replace $Z$ by a point $p t \in Y^{\tau}$ ( $Y^{\tau}$ is not empty if $Y$ is a conjugation space) and use Remark 3.2 .

We shall now apply Proposition 4.1. The pair $(Y, Z)$ being a conjugation pair by hypothesis, we must check that $(X, Y)$ is a conjugation pair. By excision, $H^{*}(X, Y)=H^{*}(D, S)$, where $D=\amalg_{\Lambda} D_{\lambda}^{2 k}$ and $S=\amalg_{\Lambda} S_{\lambda}^{2 k-1}$. One also has $H_{C}^{*}(X, Y)=H_{C}^{*}(D, S)$ and $H^{*}\left(X^{\tau}, Y^{\tau}\right)=H^{*}\left(D^{\tau}, S^{\tau}\right)$, with $D^{\tau}=\amalg_{\Lambda} D_{\lambda}^{k}$ and $S^{\tau}=\amalg_{\Lambda} S_{\lambda}^{k-1}$.

Suppose first that $\Lambda=\{\lambda\}$ has one element, so $D=D_{\lambda}$ and $S=S_{\lambda}$. As seen in Example 3.5. we get here a $H^{*}$-frame $\left(\kappa_{\lambda}, \sigma_{\lambda}\right)$ such that, if $a$ is the non-zero element of $H^{2 k}(D, S)$, the equation $r_{\lambda} \sigma_{\lambda}(a)=\kappa_{\lambda}(a) u^{k}$ holds. For the general case, one has $H^{*}(D, S)=\prod_{\lambda \in \Lambda} H^{*}\left(D_{\lambda}, S_{\lambda}\right), H_{C}^{*}(D, S)=\prod_{\lambda \in \Lambda} H_{C}^{*}\left(D_{\lambda}, S_{\lambda}\right)$, etc, and $\rho=\prod_{\lambda \in \Lambda} \rho_{\lambda}, r=\prod_{\lambda \in \Lambda} r_{\lambda}$. The homomorphisms $\sigma=\prod_{\lambda \in \Lambda} \sigma_{\lambda}$ and $\kappa=\prod_{\lambda \in \Lambda} \kappa_{\lambda}$ satisfy $r \sigma(a)=\kappa(a) u^{k}$ for all $a \in H^{2 k}(D, S)=H^{*}(D, S)$. This shows that $(D, S)$ and then $(X, Y)$ is a conjugation pair.

We then know that $(X, Y)$ and $(Y, Z)$ are conjugation pairs. By Proposition $4.1,(X, Z)$ is a conjugation pair.

\subsection{Conjugation complexes}

Let $Y$ be a space with an involution $\tau$. A space $X$ is a spherical conjugation complex relative to $Y$ if it is equipped with a filtration

$$
Y=X_{-1} \subset X_{0} \subset X_{1} \subset \cdots X=\bigcup_{k=-1}^{\infty} X_{k}
$$

where $X_{k}$ is obtained from $X_{k-1}$ by the adjunction of a collection of conjugation cells (indexed by a set $\left.\Lambda_{k}(X)\right)$. The topology on $X$ is the direct limit topology of the $X_{k}$ 's. If $Y$ is empty, we say that $X$ is a spherical conjugation complex. As in [11, the adjective "spherical" emphasizes that the collections of conjugation cells need not occur in increasing dimensions.

The involution $\tau$ on $Y$ extends naturally to an involution on $X$, still called $\tau$. The following result is a direct consequence of Proposition [5.1] and Proposition 4.6 .

Proposition 5.2 Let $X$ be a spherical conjugation complex relative to $Y$. Then the pair $(X, Y)$ is a conjugation pair. 


\subsection{Remarks and Examples}

5.3.1 Many topological properties of CW-complexes remain true for spherical conjugation complexes, using minor adaptations of the standard techniques (see e.g. [18]). For instance, a spherical conjugation complex is paracompact, by the same proof as in [18, Theorem 4.2]. Also, the product $X \times Y$ of two conjugation spaces admits a spherical conjugation complex-structure provided $X$ contains finitely many conjugation cells, or both $X$ and $Y$ contain countably many conjugation cells. For instance, one can order the elements $(p, q) \in \mathbb{N} \times \mathbb{N}$ by the lexicographic ordering in $(p+q, p)$ and construct a conjugation space $X \otimes Y$ by setting $(X \times Y)_{(p, q)}=X_{p} \times X_{q}$. If $\left(p^{\prime}, q^{\prime}\right)$ is the successor of $(p, q)$, then using that the product of a conjugation cell is a conjugation cell, one shows that $(X \times Y)_{\left(p^{\prime}, q^{\prime}\right)}$ is obtained from $(X \times Y)_{(p, q)}$ by adjunction of a collection of conjugation cells indexed by $\Lambda_{\left(p^{\prime}, q^{\prime}\right)}(X \times Y)=\Lambda_{p^{\prime}}(X) \times \Lambda_{q^{\prime}}(Y)$. There is then a $\tau$-equivariant continuous bijection $\theta: X \otimes Y \rightarrow X \times Y$. As in [18. II.5, Theorem 5.2], one shows that, under the above hypotheses, $\theta$ is an homeomorphism.

5.3.2 The usual cell decomposition of $\mathbb{C} P^{n}(n \leq \infty)$ makes the latter a spherical conjugation complex. The product of finitely many copies of $\mathbb{C} P^{\infty}$ is also a spherical conjugation complex. Here, we do not even need the preceeding remark since we are just dealing with the product of countable $\mathrm{CW}$-complexes.

Let $T$ be a torus (compact abelian group) of dimension $r$. The involution $g \mapsto g^{-1}$ induces an involution on the Milnor classifying space BT. The latter is equivariantly homotopy equivalent to a product of $r$ copies of $\mathbb{C} P^{\infty}$ and therefore is a conjugation space. The isomorphism $\kappa_{T}$ of the $H^{*}$-frame for $B T$ can be interpreted as follows.

Let $\hat{T}=\operatorname{Hom}\left(T, S^{1}\right)$ be the group of characters of $T$. We have identifications

$$
\hat{T} \approx\left[B T, \mathbb{C} P^{\infty}\right] \approx H^{2}(B T ; \mathbb{Z}) .
$$

Recall that $\hat{T}$ is a free abelian group of rank the dimension of $T$. Hence $H^{2}(B T)$ is isomorphic to $\hat{T} \otimes \mathbb{Z}_{2}$. For the 2 -torus subgroup $T_{2}$ of $T$, defined to be the elements of $T$ of order 2 , one has in the same way

$$
\operatorname{Hom}\left(T_{2}, S^{0}\right) \approx\left[B T_{2}, \mathbb{R} P^{\infty}\right] \approx H^{1}\left(B T_{2}\right),
$$

where we think of $S^{0}=\{ \pm 1\}$ as the 2-torus of $S^{1}$. The homomorphism $\hat{T} \rightarrow \operatorname{Hom}\left(T_{2}, S^{0}\right)$, which sends $\chi \in \hat{T}$ to the restriction $\chi_{2}$ of $\chi$ to $T_{2}$, produces an isomorphism $\kappa_{T}: H^{2}(B T) \rightarrow H^{1}\left(B T_{2}\right)$. Now the cohomology ring $H^{2 *}(B T)=S\left(H^{2}(B T)\right)$ is the symmetric algebra over $H^{2}(B T)$, and 
$H^{*}\left(B T_{2}\right)=S\left(H^{1}\left(B T_{2}\right)\right)$. Therefore, the above isomorphism $\kappa_{T}$ extends to a ring isomorphism $\kappa_{T}: H^{2 *}(B T) \rightarrow H^{*}\left(B T_{2}\right)$ that is functorial in $T$. Now, $B T_{2}=B T^{\tau}$, and $\kappa_{T}$ is the isomorphism in the $H^{*}$-frame of $B T$. This can be checked by choosing an isomorphism between $T$ and $\left(S^{1}\right)^{r}$, which induces a $C$ equivariant homotopy equivalence between $B T$ and $\left(\mathbb{C} P^{\infty}\right)^{r}$ and a homotopy equivalence between $B T^{\tau}$ and $\left(\mathbb{R} P^{\infty}\right)^{r}$.

5.3.3 Example 5.3.2 generalizes to complex Grassmannians, with the complex conjugation. The classical Schubert cells give the spherical conjugation complex-structure. This generalizes to the coadjoint orbits of compact semisimple Lie groups with the Chevalley involution (see Subsection 8.3), using the Bruhat-Schubert cells.

5.3.4 Conjugation complexes with 3 conjugation cells. Let $X$ be a spherical conjugation complex with three conjugation cells, in dimension $0,2 k$ and $2 l \geq$ $2 k$. Then, $X$ is obtained by attaching a conjugation cell $D^{2 l}$ to the conjugation sphere $\Sigma^{2 k}$ (see Example [3.6). The $C$-equivariant homotopy type of $X$ is determined by the class of the attaching map $\alpha \in \pi_{2 l-1}^{\tau}\left(\Sigma^{2 k}\right)$, the equivariant homotopy group of $\Sigma^{2 k}$ (the homotopy classes of equivariant maps from $\Sigma^{2 l-1}$ to $\Sigma^{2 k}$ ). We note $X=X_{\alpha}$. Forgetting the $C$-equivariance and restricting to the fixed point sets gives a homomorphism

$$
\Phi_{l, k}: \pi_{2 l-1}^{\tau}\left(\Sigma^{2 k}\right) \rightarrow \pi_{2 l-1}\left(S^{2 k}\right) \times \pi_{l-1}\left(S^{k}\right) .
$$

In the case $k=1$ and $l=2$, this gives

$$
\Phi:=\Phi_{2,1}: \pi_{3}^{\tau}\left(\Sigma^{2}\right) \rightarrow \pi_{3}\left(S^{2}\right) \times \pi_{1}\left(S^{1}\right)=\mathbb{Z} \times \mathbb{Z} .
$$

Observe that the equivariant homotopy type of $X_{\alpha}$ and of $X_{\beta}$ are distinct if $\Phi(\alpha) \neq \Phi(\beta)$. Indeed, let $\Phi(\alpha)=(p, q)$. If $a \in H^{2}(X ; \mathbb{Z})$ and $b \in H^{4}(X ; \mathbb{Z})$ are the natural generators, then $a^{2}=p b$ (see, e.g. [25. §9.5, Theorem 3]). Moreover $H^{1}\left(X^{\tau} ; \mathbb{Z}\right)=\mathbb{Z}_{q}$. Note that since $X$ is a conjugation space, one has $H^{1}\left(X^{\tau}\right)=\mathbb{Z}_{2}$, which shows that $q$ must be even.

Now, it is easy to see that the Hopf map $h: \Sigma^{3} \rightarrow \Sigma^{2}$ is $C$-equivariant; as $\Phi(h)=(1,2)$ is of infinite order, this shows that there are infinitely many $C$-equivariant homotopy types of spherical conjugation complexes with three conjugation cells, in dimension 0,2 and 4 .

\subsection{Equivariant fiber bundles over spherical conjugation com- plexes}

Let $G$ be a topological group together with an involution $\sigma$ which is an automorphism of $G$. Let $(B, \tau)$ be a space with involution. By a $(\sigma, G)$-principal 
bundle we mean a (locally trivial) $G$-principal bundle $p: E \rightarrow B$ together with an involution $\tilde{\tau}$ on $E$ satisfying $p \circ \tilde{\tau}=\tau \circ p$ and $\tilde{\tau}(z \cdot g)=\tilde{\tau}(z) \cdot \sigma(g)$ for all $z \in E$ and $g \in G$. Following the terminology of [26, p. 56], a $(\sigma, G)$-principal bundle is a $(C, \check{\sigma}, G)$-bundle, where $\check{\sigma}: C \rightarrow G$ is the homomorphism sending the generator of $C$ to $\sigma$.

Let $F$ be a space together with an involution $\tau$ and a left $G$-action. We say that the involution $\tau$ and the $G$-action are compatible if $\tau(g y)=\sigma(g) \tau(y)$. This means that the $G$-action extends to an action of the semi-direct product $G^{\times}=G \rtimes C$.

Let $p: E \rightarrow B$ be a $(\sigma, G)$-principal bundle. Let $(F, \tau)$ be a space with involution together with a compatible $G$-action. The space $E \times_{G} F$ inherits an involution (also called $\tau$ ) and the associated bundle $E \times{ }_{G} F \rightarrow B$, with fiber $F$, is a $\tau$-equivariant locally trivial bundle.

Proposition 5.3 Suppose that $G$ is a compact Lie group, that $F$ is a conjugation space and that $B$ is a spherical conjugation complex. Then $E \times{ }_{G} F$ is a conjugation space.

Proof Suppose first that $B=D$ is a conjugation cell, with boundary $S$. Then $E$ is compact and, by [26, Ch. 1, Proposition 8.10], $p$ is a locally trivial $(C, \tilde{\sigma}, G)$-bundle. This means that there exists an open covering $\mathcal{U}$ of $B$ by $C$-invariant sets such that for each $U \in \mathcal{U}$ the bundle $p^{-1}(U) \rightarrow U$ is induced by a $(\sigma, G)$-principal bundle over a $C$-orbit (namely one point or two points). Since the quotient space $C \backslash D$ is compact, the coverings $\mathcal{U}$ admits a partition of unity by $C$-invariant maps. Together these imply that the $(\sigma, G)$-bundle $p$ is induced from a universal $(C, \tilde{\sigma}, G)$-bundle by a $C$-equivariant map from $D$ to some classifying space and $C$-homotopic maps induce isomorphic $(C, \tilde{\sigma}, G)$ bundles [26. Ch.1, Theorem 8.12 and 8.15]. The cell $D$ is $C$-contractible, which implies that $E=D \times G$ and $E \times{ }_{G} F=D \times F$, with the product involution. By Proposition 4.5, the pair $\left(E, E_{\mid S}\right)$ is a conjugation pair.

This enables us to prove Proposition 5.3 by induction on the $n$-stage $B_{n}$ of the construction of $B$ as a spherical conjugation complex. Let $Z_{n}=p^{-1}\left(B_{n}\right) \times_{G} F$. As $B_{0}$ is discrete, $Z_{0}$ is the disjoint union of copies of $F$ and is then a conjugation space. Suppose by induction that $Z_{n-1}$ is a conjugation space. The above argument shows that $\left(Z_{n}, Z_{n-1}\right)$ is a conjugation pair. Using Proposition 4.1, one deduces that $Z_{n}$ is a conjugation space. Therefore, $Z_{n}$ is a conjugation space for all $n \in \mathbb{N}$. By Proposition 4.6 this implies that $E \times_{G} F=\lim _{\rightarrow} Z_{n}$ is a conjugation space. 
Remark 5.4 An analogous argument also gives a relative version of Proposition 5.3 for pairs of bundles over $X$, with a conjugation pair of fibers $\left(F, F_{0}\right)$. The same remains true for a bundle over a relative spherical conjugation complex.

\section{Conjugate-equivariant complex bundles}

\subsection{Definitions}

Let $(X, \tau)$ be a space with an involution. A $\tau$-conjugate-equivariant bundle (or, briefly, a $\tau$-bundle) over $X$ is a complex vector bundle $\eta$, with total space $E=\mathbb{E}(\eta)$ and bundle projection $p: E \rightarrow X$, together with an involution $\hat{\tau}: E \rightarrow E$ such that $p \circ \hat{\tau}=\tau \circ p$ and $\hat{\tau}$ is conjugate-linear on each fiber: $\hat{\tau}(\lambda x)=\bar{\lambda} \hat{\tau}(x)$ for all $\lambda \in \mathbb{C}$ and $x \in E$. Atiyah was the first to study $\tau$-bundles [2]. He called them "real bundles" and used them to define $K R$ theory.

Let $P \rightarrow X$ be a $(\sigma, U(r))$-principal bundle in the sense of Subsection 5.4, with $\sigma: U(r) \rightarrow U(r)$ being the complex conjugation. Then, the associated bundle $P \times_{U(r)} \mathbb{C}^{r}$, with $\mathbb{C}^{r}$ equipped with the complex conjugation, is a $\tau$-bundle and any $\tau$-bundle is of this form. It follows that if $p: E \rightarrow X$ be a $\tau$-bundle $\eta$ of rank $r$ and if $E^{\hat{\tau}}$ is the fixed point set of $\hat{\tau}$, then $p: E^{\hat{\tau}} \rightarrow X^{\tau}$ is a real vector bundle $\eta^{\tau}$ of rank $r$ over $X^{\tau}$.

Examples of $\tau$-bundle include the canonical complex vector bundle over $B U(r)$ or over the complex Grassmannians. Note that a bundle induced from a $\tau$ bundle by a $C$-equivariant map is a $\tau$-bundle.

Proposition 6.1 Let $\eta$ be a $\tau$-bundle of rank $r$ over a space with involution $(X, \tau)$. If $X$ is paracompact, then $\eta$ is induced from the universal bundle by a $C$-equivariant map from $X$ into $B U(r)$. Moreover, two $C$-equivariant map which are $C$-homotopic induce isomorphic $\tau$-bundles.

Proof It is equivalent to prove the corresponding statement of Proposition 6.1 for $(\sigma, U(r))$-bundles. Let $p: P \rightarrow X$ be a $(\sigma, U(r))$-bundle. As $X$ is paracompact and $U(r)$ is compact, the total space $P$ is paracompact. Therefore, by [26. Ch. 1, Proposition 8.10], $p$ is a locally trivial $(\sigma, U(r))$-bundle, meaning that there exists an open covering $\mathcal{V}$ of $X$ by $C$-invariant sets such that for each $V \in \mathcal{V}$ the bundle $p^{-1}(V) \rightarrow V$ is induced by a $(\sigma, G)$-principal bundle $q: q_{\mathcal{O}} \rightarrow \mathcal{O}$ over a $C$-orbit $\mathcal{O}$. When $\mathcal{O}$ consists of one point $a$, one can identify 
$Q_{\mathcal{O}}$ with $U(r)$ such $\tilde{\tau}(\gamma)=\bar{\gamma}$. For a free orbit $\mathcal{O}=\{a, b\}$, one can identify $Q_{\mathcal{O}}$ with $\mathcal{O} \times U(r)$ such that $\tilde{\tau}(a, \gamma)=(b, \bar{\gamma})$ and $\tilde{\tau}(b, \gamma)=(a, \bar{\gamma})$. Using these, one gets a family of $U(r)$-equivariant maps $\left\{\varphi_{V}: p^{-1}(V) \rightarrow U(r) \mid V \in \mathcal{V}\right\}$ such that

$$
\varphi_{V} \circ \tau(z)=\overline{\varphi_{V}(z)}
$$

for all $V \in \mathcal{V}$. The quotient space $C \backslash X$ is also paracompact. Therefore, the coverings $\mathcal{V}$ admits a locally finite partition of the unity $\mu_{V}, V \in \mathcal{V}$, by $C$ invariant maps. Using $\left\{\varphi_{V}, \mu_{V} \mid V \in \mathcal{V}\right\}$, we can perform the classical Milnor construction of a map $f: X \rightarrow B U(r)$ inducing $p$. Because of Equation (6.1), $f$ is $C$-equivariant. The last statement of Proposition 6.1 is a direct consequence of [26. Ch. 1, Theorem 8.12 and 8.15].

Corollary 6.2 Let $\eta$ be a $\tau$-bundle over a conjugation cell. Then, the total space of disk bundle $\mathbb{D}(\eta)$ is a conjugation cell.

Proof As a conjugation cell is $C$-contractible, Proposition 6.1 implies that $\eta$ is a product bundle. We then use that the product of two conjugation cells is a conjugation cell.

Remark 6.3 Pursuing in the way of Proposition 6.1 one can prove that the set of isomorphism classes of $\tau$-bundles of rank $r$ over a paracompact space $X$ is in bijection with the set of $C$-equivariant homotopy classes of $C$-equivariant maps from $X$ to $B U(r)$.

\subsection{Thom spaces}

Proposition 6.4 Let $\eta$ be a $\tau$-bundle over a conjugation space $X$. Then the total space $\mathbb{D}(\eta)$ of the disk bundle of $\eta$ and the total space $\mathbb{S}(\eta)$ of the sphere bundle of $\eta$ form a conjugation pair $(\mathbb{D}(\eta), \mathbb{S}(\eta))$.

Proof Let $\mathbb{E}(\eta) \rightarrow X$ be the bundle projection and let $r$ be the rank of $\eta$. Performing the Borel construction $\mathbb{E}(\eta)_{C} \rightarrow X_{C}$ gives a complex bundle $\eta_{C}$ of rank $r$ over $X_{C}$ and $\eta$ is induced from $\eta_{C}$ by the map $X \rightarrow X_{C}$. The following diagrams, in which the letters $\mathcal{T}$ denote the Thom isomorphisms, show how to define $\bar{\sigma}$ and $\bar{\kappa}$.

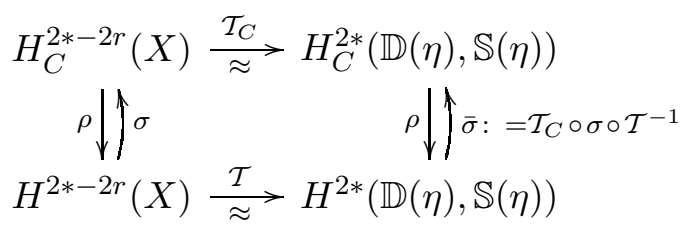




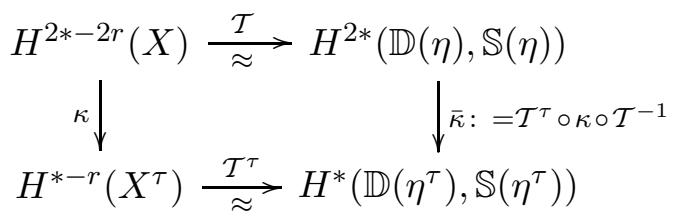

Consider also the following commutative diagram, where the vertical arrows are restriction to a fiber.

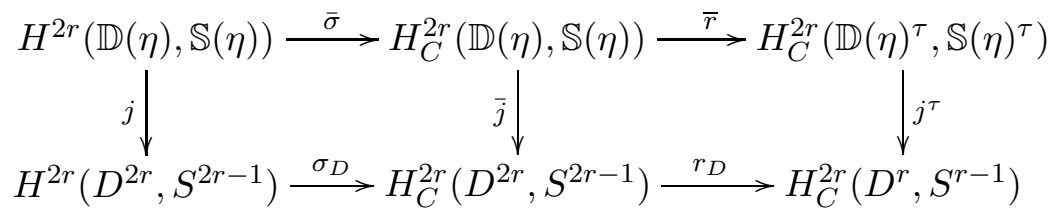

It remains to prove the conjugation equation. Let $\operatorname{Thom}(\eta) \in H^{2 r}(\mathbb{D}(\eta), \mathbb{S}(\eta))$ be the Thom class of $\eta$. By definition of $\bar{\sigma}$, one has $\bar{\sigma}(\operatorname{Thom}(\eta))=\operatorname{Thom}\left(\eta_{C}\right)$. Observe that $D^{2 r}$ is a conjugation cell and $\bar{j}\left(\operatorname{Thom}\left(\eta_{C}\right)\right)=\sigma_{D}\left(\left[D^{2 r}, S^{2 r-1}\right]\right)$. Therefore

$$
r \circ \sigma_{D} \circ j(\operatorname{Thom}(\eta))=\kappa_{D^{2 r}}\left(\left[D^{2 r}, S^{2 r-1}\right]\right) u^{r}=\left[D^{r}, S^{r-1}\right] u^{r} .
$$

But $r \circ \sigma_{D} \circ j=j^{\tau} \circ \bar{r}_{\circ} \bar{\sigma}$ and the preimage under $j^{\tau}$ of $\left[\left(D^{r}, S^{r-1}\right)\right]$ is $\operatorname{Thom}\left(\eta^{\tau}\right)$. By Lemma 2.2, the kernel of $j^{\tau} H_{C}^{2 r}\left(\mathbb{D}(\eta)^{\tau}, \mathbb{S}(\eta)^{\tau}\right) \rightarrow H_{C}^{2 r}\left(D^{r}, S^{r-1}\right)$ is of type $\ell t_{r}$. Therefore, one has

$$
\bar{r} \circ \bar{\sigma}(\operatorname{Thom}(\eta))=\bar{r}\left(\operatorname{Thom}\left(\eta_{C}\right)\right)=\operatorname{Thom}\left(\eta^{\tau}\right) u^{r}+\ell t_{r} .
$$

Using Equation (6.3), one has, for $x \in H^{2 k+2 r}(\mathbb{D}(\eta), \mathbb{S}(\eta))$ :

$$
\begin{aligned}
\bar{r} \circ \bar{\sigma}(x) & =\bar{r} \circ \mathcal{T}_{C} \circ \sigma \circ \mathcal{T}^{-1}(x)=\bar{r}\left(\operatorname{Thom}\left(\eta_{C}\right) \cdot \sigma \circ \mathcal{T}^{-1}(x)\right) \\
& =\bar{r}\left(\operatorname{Thom}\left(\eta_{C}\right) \cdot r \circ \sigma \circ \mathcal{T}^{-1}(x)\right) \\
& =\left(\operatorname{Thom}\left(\eta^{\tau}\right) u^{r}+\ell t_{r}\right)\left(\kappa\left(\mathcal{T}^{-1}(x)\right) u^{k}+\ell t_{k}\right) \\
& =\operatorname{Thom}\left(\eta^{\tau}\right) \kappa\left(\mathcal{T}^{-1}(x)\right) u^{k+r}+\ell t_{k+r}=\bar{\kappa}(x) u^{k+r}+\ell t_{k+r} .
\end{aligned}
$$

Remark 6.5 The pair $(\mathbb{D}(\eta), \mathbb{S}(\eta))$ is cohomologically equivalent to the pair $(\mathbb{D}(\eta) / \mathbb{S}(\eta), p t)$ and $\mathbb{D}(\eta) / \mathbb{S}(\eta)$ is the Thom space of $\eta$. Using Remark 3.2 Proposition 6.4 says that if $\eta$ is a $\tau$-bundle over a conjugation space, then the Thom space of $\eta$ is a conjugation space.

Remark 6.6 By the definition of $\bar{\kappa}: H^{2 *}(\mathbb{D}(\eta), \mathbb{S}(\eta)) \rightarrow H^{*}\left(\mathbb{D}\left(\eta^{\tau}\right), \mathbb{S}\left(\eta^{\tau}\right)\right)$, one has $\left.\bar{\kappa}(\operatorname{Thom}(\eta))=\operatorname{Thom}\left(\eta^{\tau}\right)\right)$. The inclusion $(\mathbb{D}(\eta), \emptyset) \subset(\mathbb{D}(\eta), \mathbb{S}(\eta))$ is a $C$-equivariant map between conjugation pairs and $\mathbb{D}(\eta)$ is $C$-homotopy equivalent to $X$. The induced homomorphisms on cohomology $i: H^{2 r}(\mathbb{D}(\eta), \mathbb{S}(\eta)) \rightarrow$ 
$H^{2 r}(X)$ and $i^{\tau}: H^{r}\left(\mathbb{D}\left(\eta^{\tau}\right), \mathbb{S}\left(\eta^{\tau}\right)\right) \rightarrow H^{r}\left(X^{\tau}\right)$ send the Thom classes Thom $(\eta)$ and $\operatorname{Thom}\left(\eta^{\tau}\right)$ to the Euler classes $e(\eta)$ and $e\left(\eta^{\tau}\right)$. By naturality of the $H^{*}$ frames, we deduce that, for any conjugate equivariant bundle $\eta$ over a conjugation space $X$, one has $\kappa(e(\eta))=e\left(\eta^{\tau}\right)$. This will be generalized in Proposition 6.8

We finish this subsection with the analogue of Proposition 6.4 for spherical conjugation complexes.

Proposition 6.7 Let $\eta$ be a $\tau$-bundle over a spherical conjugation complex $X$. Then, $\mathbb{D}(\eta)$ is a spherical conjugation complex relative to $\mathbb{S}(\eta)$.

Proof Let $X$ be obtained from $Y$ by attaching a collection of conjugation cells of dimension $2 k$, indexed by a set $\Lambda$. Let $D=\amalg_{\Lambda} D_{\lambda}^{2 k}$ and $S=\amalg_{\Lambda} S_{\lambda}^{2 k-1}$ $(\lambda \in \Lambda)$. Let $\pi=\pi_{D} \amalg \pi_{Y}: D \amalg Y \rightarrow X$ be the natural projection. Then $\mathbb{D}(\eta)$ is obtained from $\mathbb{D}\left(\pi_{Y}^{*} \eta\right) \cup \mathbb{S}(\eta)$ by attaching $\mathbb{D}\left(\pi_{D}^{*} \eta\right)$. By Corollary 6.2, $\mathbb{D}\left(\pi_{D_{\lambda}}^{*} \eta\right)$ is a conjugation cell of dimension $2 k+2 r$, where $r$ is the complex rank of $\eta$. Therefore, $\mathbb{D}(\eta)$ is obtained from $\mathbb{D}\left(\pi_{Y}^{*} \eta\right) \cup \mathbb{S}(\eta)$ by attaching a collection of conjugation cells of dimension $2 k+2 r$. This proves Proposition 6.7

\subsection{Characteristic classes}

If $\eta$ be a $\tau$-bundle over a space with involution $X$, we denote by $c(\eta) \in H^{2 *}(X)$ the $(\bmod 2)$ total Chern class of $\eta$ and by $w\left(\eta^{\tau}\right) \in H^{*}\left(X^{\tau}\right)$ the total StiefelWhitney class of $\eta^{\tau}$. The aim of this section is to prove the following:

Proposition 6.8 Let $\eta$ be a $\tau$-bundle over a spherical conjugation complex $X$. Then $\kappa(c(\eta))=w\left(\eta^{\tau}\right)$.

Proof Let $q: \mathbb{P}(\eta) \rightarrow X$ be the projective bundle associated to $\eta$, with fiber $\mathbb{C} P^{r-1}$. The conjugate-linear involution $\hat{\tau}$ on $\mathbb{E}(\eta)$ descends to an involution $\tilde{\tau}$ on $\mathbb{P}(\eta)$ for which the projection $q$ is equivariant. One has $\mathbb{P}(\eta)^{\tilde{\tau}}=\mathbb{P}\left(\eta^{\tau}\right)$, the projective bundle associated to $\eta^{\tau}$, with fiber $\mathbb{R} P^{r-1}$. We also call $q: \mathbb{P}\left(\eta^{\tau}\right) \rightarrow$ $X^{\tau}$ the restriction of $q$ to $\mathbb{P}\left(\eta^{\tau}\right)$.

As $q$ is equivariant, the induced complex vector bundle $q^{*} \eta$ is a $\tilde{\tau}$-bundle with $\mathbb{E}\left(q^{*} \eta\right)^{\tau}=\mathbb{E}\left(q^{*} \eta^{\tau}\right)$. Recall that $q^{*} \eta$ admits a canonical line subbundle $\lambda_{\eta}$ : a point of $\mathbb{E}\left(\lambda_{\eta}\right)$ is a couple $(L, v) \in \mathbb{P}(\eta) \times \mathbb{E}(\eta)$ with $v \in L$. The same formula holds for $\eta^{\tau}$, giving a real line subbundle $\lambda_{\eta^{\tau}}$ of $q^{*} \eta^{\tau}$. Moreover, $\hat{\tau}(v) \in \tau(L)$ and thus $\lambda_{\eta}$ is a $\tilde{\tau}$-conjugate-equivariant line bundle over $\mathbb{P}(\eta)$. 
Again, $\mathbb{E}\left(\lambda_{\eta}\right)^{\tau}=\mathbb{E}\left(\lambda_{\eta^{\tau}}\right)$. The quotient bundle $\eta_{1}$ of $\eta$ by $\lambda_{\eta}$ is also a $\tilde{\tau}$-bundle over $\mathbb{P}(\eta)$ and $q^{*} \eta$ is isomorphic to the equivariant Whitney sum of $\lambda_{\eta}$ and $\eta_{1}$.

By Proposition $5.3 \mathbb{P}(\eta)$ is a conjugation space. Denote by $(\tilde{\kappa}, \tilde{\sigma})$ its $H^{*}$-frame. By Remark 6.6, one has $\tilde{\kappa}\left(c_{1}\left(\lambda_{\eta}\right)\right)=w_{1}\left(\lambda_{\eta^{\tau}}\right)$. As $\tilde{\kappa}$ is a ring isomorphism, one has $\tilde{\kappa}\left(c_{1}\left(\lambda_{\eta}\right)^{k}\right)=w_{1}\left(\lambda_{\eta^{\tau}}\right)^{k}$ for each integer $k$.

By [15. Chapter 16,2.6], we have in $H^{2 *}(\mathbb{P}(\eta))$ the equation

$$
c_{1}\left(\lambda_{\eta}\right)^{r}=\sum_{i=1}^{r} q^{*}\left(c_{i}(\eta)\right) c_{1}\left(\lambda_{\eta}\right)^{r-i} .
$$

and, in $H^{*}\left(\mathbb{P}\left(\eta^{\tau}\right)\right)$,

$$
w_{1}\left(\lambda_{\eta^{\tau}}\right)^{r}=\sum_{i=1}^{r} q^{*}\left(w_{i}\left(\eta^{\tau}\right)\right) w_{1}\left(\lambda_{\eta^{\tau}}\right)^{r-i} .
$$

As $\tilde{\kappa}\left(c_{1}\left(\lambda_{\eta}\right)\right)=w_{1}\left(\lambda_{\eta^{\tau}}\right)$ and $\tilde{\kappa} \circ q^{*}=q^{*} \circ \kappa$, applying $\tilde{\kappa}$ to Equation (6.4) and using Equation (6.5) gives

$$
\sum_{i=1}^{r} q^{*}\left(\kappa\left(c_{i}(\eta)\right)\right) w_{1}\left(\lambda_{\eta^{\tau}}\right)^{r-i}=\sum_{i=1}^{r} q^{*}\left(w_{i}\left(\eta^{\tau}\right)\right) w_{1}\left(\lambda_{\eta^{\tau}}\right)^{r-i} .
$$

By the Leray-Hirsch theorem, $H^{*}\left(\mathbb{P}\left(\eta^{\tau}\right)\right)$ is a free $H^{*}\left(X^{\tau}\right)$-module with basis $w_{1}\left(\lambda_{\eta}\right)^{k}$ for $k=1, \ldots, r-1$, and $q^{*}$ is injective. Therefore, Equation (6.6) implies Proposition 6.8.

Remark 6.9 By Proposition 6.1, it would be enough to prove Proposition 6.8 for the canonical bundle over the Grassmannian. This can be done via the Schubert calculus (see [21, Problem 4-D, p. 171, and §6]). Such an argument proves Proposition 6.8 for $X$ a paracompact conjugation space.

\section{Compatible torus actions}

Let $X$ be a space together with an involution $\tau$. Suppose that a torus $T$ acts continuously on $X$. We say that the involution $\tau$ is compatible with this torus action if $\tau(g \cdot x)=g^{-1} \cdot \tau(x)$ for all $g \in T$ and $x \in X$. It follows that $\tau$ induces an involution on on the fixed point set $X^{T}$. Moreover, the 2 -torus subgroup $T_{2}$ of $T$, defined to be the elements of $T$ of order 2 , acts on $X^{\tau}$. The involution and the $T$-action extend to an action of the semi-direct product $T^{\times}=T \rtimes C$, where $C$ acts on $T$ by $\tau \cdot g=g^{-1}$. 
When a group $H$ acts on $X$, we denote by $X_{H}$ the Borel construction of $X$. Observe that if $T^{\times}$as above acts on $X$, then the diagonal action of $C$ on $E T \times X$ descends to an action of $C$ on $X_{T}$.

Lemma 7.1 Let $X$ be a space together with a continuous action of $T^{\times}$. Then $X_{T^{\times}}$has the homotopy type of $\left(X_{T}\right)_{C}$.

Proof $X_{T}$ has the $C$-equivariant homotopy type of the quotient $T \backslash E T^{\times} \times X$, where $T$ acts on $E T^{\times} \times X$ by $g \cdot(w, x)=\left(w g^{-1}, g x\right)$. The formula $\tau \cdot(w, x)=$ $(w \tau, \tau(x))$ then induces a $C$-action on $X_{T}$ which is free. Therefore, $\left(X_{T}\right)_{T^{\times}}=$ $C \backslash X_{T}=X_{T \times}$.

The particular case of $X=p t$ in Lemma 7.1 gives the following:

Corollary $7.2 B T^{\times} \simeq B T_{C}$.

Lemma $7.3\left(X_{T}\right)^{\tau}=\left(X^{\tau}\right)_{T_{2}}$.

Proof Let $H$ be a group acting continuously on a space $Y$. Recall that elements of the infinite joint $E H$ are represented by sequences $\left(t_{i} h_{i}\right)(i \in \mathbb{N})$ with $h_{i} \in H$ and $t_{i} \in[0,1]$, almost all vanishing, with $\sum t_{i}=1$. Under the right diagonal action of $H$ on $E H$, each $\left(t_{i} h_{i}\right)$ is equivalent to a unique element $\left(t_{i} \tilde{h}_{i}\right)$ for which $\tilde{h}_{j}=I$, the unit element of $H$, where $j$ is the minimal integer $k$ for which $t_{k} \neq 0$. Therefore, each class in $B H=E H / H$ has a unique such representative which we call minimal. In the same way, each class in $Y_{H}$ has a unique minimal representative $(w, y) \in E H \times Y$ for which $w$ is minimal.

One easily check that there is a commutative diagram:

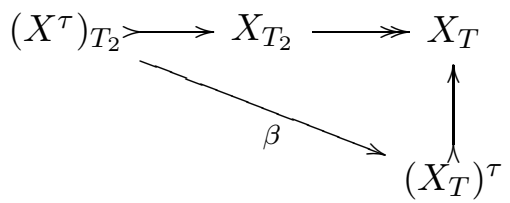

Working with minimal representatives in $\left(X^{\tau}\right)_{T_{2}}$, we see that the natural map $\left(X^{\tau}\right)_{T_{2}} \rightarrow X_{T}$ is injective. Hence, $\beta$ is injective. Let $(w, x) \in E T \times X$ with $w=\left(t_{i} z_{i}\right)$ minimal. Then, $\tau(w, x)$ is also a minimal representative. If $\tau(w, x)=$ $(w, x)$ in $X_{T}$, this implies that $\tau(x)=x$ and $z_{i}^{-1}=z_{i}$, that is $z_{i} \in T_{2}$ (when $\left.t_{i} \neq 0\right)$. This proves that $\beta$ is surjective. 
Example 7.4 Let $X=S^{1} \subset \mathbb{C}$ with the complex conjugation as involution, and $T=S^{1}$ acting on $X$ by $g \cdot z=g^{2} z$. Then, $X^{\tau}=S^{0}$ on which $T_{2}$ acts trivially, so $\left(X^{\tau}\right)_{T_{2}}=B T_{2} \times S^{0}$. On the other hand, $X$ is a $T$-orbit so $X_{T}=E T / T_{2}$. The space $E T / T_{2}$ has the homotopy type of $B T_{2}$ but $\left(E T / T_{2}\right)^{\tau}$ has two connected components, both homeomorphic to $B T_{2}$. One is the image of $(E T)^{\tau}=E T_{2}$ and is equal to $\beta\left(B T_{2} \times\{1\}\right)$. The other is the image of $\left\{\left(t_{j} h_{j}\right) \mid h_{j}= \pm i\right\}$ and is equal to $\beta\left(B T_{2} \times\{-1\}\right)$.

The main result of this section is the following:

Theorem 7.5 Let $(X, Y)$ be a conjugation pair together with a compatible action of a torus $T$. Then, the involution induced on $\left(X_{T}, Y_{T}\right)$ is a conjugation.

Proof Assume first that $Y=\emptyset$. The universal bundle $p: E T \rightarrow B T$ is a $(T, \sigma)$-principal bundle in the sense of Subsection 5.4. with $\sigma(g)=g^{-1}$, and $X_{T} \rightarrow B T$ is the associated bundle with fiber $X$. As $B T$ is a conjugation space (see Remark 5.3.2 in Subsection [5.3), the space $X_{T}$ is a conjugation space by Proposition 5.3. When $Y$ is not empty, we use Remark [5.4.

Using Lemma 7.3. one gets the following corollary of Theorem 7.5 .

Corollary 7.6 Let $X$ be a space together with an involution and a compatible $T$-action. Then, there is a ring isomorphism

$$
\bar{\kappa}: H_{T}^{2 *}(X) \stackrel{\approx}{\longrightarrow} H_{T_{2}}^{*}\left(X^{\tau}\right) .
$$

We end this section with a result that will be used in Section 8 , Let $\eta$ be a $T$-equivariant $\tau$-bundle over a space with involution $X$. Precisely, $\eta$ is a $\tau$ bundle over $X$ and there is a $\hat{\tau}$-compatible $T$-action on $\mathbb{E}(\eta)$, over the identity of $X$, which is $\mathbb{C}$-linear on each fiber. Let $r$ be the complex rank of $\eta$. The $T$-Borel construction on $\mathbb{E}(\eta) \rightarrow X$ produces a complex vector bundle $\eta_{T}$ of rank $r$ over $X_{T}$. One checks that the involution induced on $\mathbb{E}\left(\eta_{T}\right)=\mathbb{E}(\eta)_{T}$ makes $\eta_{T}$ a $\tau$-bundle (the letter $\tau$ also denotes here the involution induced on $\left.X_{T}=B T \times X\right)$. For a $T^{\times}$-invariant Riemannian metric on $\eta$, the spaces $\mathbb{D}(\eta)$ and $\mathbb{S}(\eta)$ are $T^{\times}$-invariant.

Proposition 7.7 Let $\eta$ be a $T$-equivariant $\tau$-bundle over a conjugation space $X$. Then the pair $\left(\mathbb{D}(\eta)_{T}, \mathbb{S}(\eta)_{T}\right)$ is a conjugation space.

Proof As the Riemannian metric is $T^{\times}$-invariant, one has $\mathbb{D}(\eta)_{T}=\mathbb{D}\left(\eta_{T}\right)$ and $\mathbb{S}(\eta)_{T}=\mathbb{S}\left(\eta_{T}\right)$. By Theorem 7.5, the base space $B T \times X$ of $\eta_{T}$ is a conjugation space. Proposition 7.7 then follows from Proposition 6.4 


\section{Hamiltonian manifolds with anti-symplectic invo- lutions}

\subsection{Preliminaries}

Let $M$ be a compact symplectic manifold equipped with a Hamiltonian action of a torus $T$. Let $\tau$ be a smooth anti-symplectic involution on $M$ compatible with the action of $T$ (see Section [7). Thus, the semi-direct group $T^{\times}:=T \rtimes C$ acts on $M$. Moreover, if it is non-empty, $M^{\tau}$ is a Lagrangian submanifold, called the real locus of $M$. For general work on such involutions together with a Hamiltonian group action, see [8] and 22].

We know that the symplectic manifold $(M, \omega)$ admits an almost Kaehler structure calibrated by $\omega$. That is, there is an almost complex structure $J \in$ End $T M$ together with a Hermitian metric $h$ whose imaginary part is $\omega$ (see [3. $\S 1.5] ; J$ and $h$ determine each other). These structures form a convex set and by averaging, we can find an almost complex structure whose Hermitian metric $\tilde{h}$ is $T$-invariant. Now, the Hermitian metric

$$
h(v, w):=\frac{1}{2}(\tilde{h}(v, w)+\overline{\tilde{h}((T \tau(v), T \tau(w))})
$$

is still $T$-invariant and satisfies $h(T \tau(v), T \tau(w))=\overline{h(v, w)}$. We suppose that the symplectic manifold $(M, \omega)$ is equipped with such an almost Kaehler structure $(J, h)$ calibrated by $\omega$, which we call a $T^{\times}$-invariant almost Kaehler structure.

Let $\Phi: M \rightarrow \mathfrak{t}^{*}$ be a moment map for the Hamiltonian torus action, where $\mathfrak{t}$ denotes the Lie algebra of $T$ and $\mathfrak{t}^{*}$ denotes its vector space dual. Evaluating $\Phi$ on a generic element $\xi$ of $\mathfrak{t}$ yields a real Morse-Bott function $\Phi^{\xi}(x)=\Phi(x)(\xi)$ whose critical point set is $M^{T}$. Suppose $F$ is a connected component of $M^{T}$. By [3, $\S$ III.1.2], $F$ is an almost Kaehler (in particular symplectic) submanifold of $M$. If $F^{\tau} \neq \emptyset$, then $F$ is preserved by $\tau: \tau(F)=F$.

Let $\nu(F)$ be the normal bundle to $F$, seen as the orthogonal complement of $T F$. The bundle $\nu(F)$ is then a complex vector bundle. By $T^{\times}$-invariance of the Hermitian metric, $\nu(F)$ admits a $\mathbb{C}$-linear $T$-action and $\tau: F \rightarrow F$ is covered by an $\mathbb{R}$-linear involution $\hat{\tau}$ of the total space $\mathbb{E}(\nu(F))$ which is compatible with the $T$-action. Moreover, $\nu(F)$ inherits a Hermitian metric $h$ whose imaginary

part is the symplectic form $\omega$. Let $x \in F$. For $v \in \mathbb{E}_{x}(\nu(F)), w \in \mathbb{E}_{\tau(x)}(\nu(F))$ and $\lambda \in \mathbb{C}$, one has

$$
\begin{aligned}
h(\hat{\tau}(\lambda v), w) & =\overline{h(\lambda v, \hat{\tau}(w))}=\bar{\lambda} \overline{h(v, \hat{\tau}(w))} \\
& =\bar{\lambda} h(\hat{\tau}(v), w)=h(\bar{\lambda} \hat{\tau}(v), w) .
\end{aligned}
$$


This shows that $\nu(F)$ is a $\tau$-bundle.

Let us decompose $\nu(F)$ into a Whitney sum of $\chi$-weight bundles $\nu^{\chi}(F)$ for $\chi \in \hat{T}$, the group of smooth homomorphisms from $T$ to $S^{1}$. Recall that the latter is free abelian of rank the dimension of $T$. We call $\nu^{\chi}(F)$ an isotropy weight bundle. Since the $T$-action on $\nu\left(M^{T}\right)$ is compatible with $\hat{\tau}$, the isotropy weight bundles are preserved by $\hat{\tau}$ and are thus $\tau$-bundles. Consequently, the negative normal bundle $\nu^{-}(F)$, which is the Whitney sum of those $\nu^{\chi}(F)$ for which $\Phi^{\xi}(\chi)<0$, is a $\tau$-bundle.

Of course $M^{T} \subset M^{T_{2}}$. The case where this inclusion is an equality will be of interest.

Lemma 8.1 The following conditions are equivalent:

(i) $M^{T}=M^{T_{2}}$.

(ii) $M^{\tau} \cap M^{T}=\left(M^{\tau}\right)^{T_{2}}$.

(iii) for each $x \in M^{T}$, there is no non-zero weight $\chi \in \hat{T}$ of the isotropy representation of $T$ at $x$ such that $\chi \in 2 \cdot \hat{T}$.

Proof If (ii) is true, then

$$
M^{\tau} \cap M^{T} \subset\left(M^{\tau}\right)^{T_{2}}=M^{\tau} \cap M^{T_{2}}=M^{\tau} \cap M^{T},
$$

which implies (i).

Each $x \in M^{T}$ has a $T^{\times}$-equivariant neighborhood $U_{x}$ on which the $T^{\times}$-action is conjugate to a linear action. The three conditions are clearly equivalent for a linear action, so Condition (i) or (ii) implies (iii).

We now show by contradiction that (iii) implies $(i i)$. Suppose that $(i i)$ does not hold: that is, there exists $x \in M^{T_{2}}$ with $x \notin M^{T}$. Let $\Phi_{t}^{\xi}$ be the gradient flow of $\Phi^{\xi}$. Then $\Phi_{t}^{\xi}$ is a $T^{+}$-equivariant diffeomorphism of $M$. Thus, $\Phi_{t}^{\xi}(x)$ has the same property of $x$ but, if $t$ is large enough, $\Phi_{t}^{\xi}(x)$ will belong to $U_{x}$ for some $x \in M^{T}$. This contradicts (iii).

Lemma 8.2 Let $M$ be a compact symplectic manifold equipped with a Hamiltonian action of a torus $T$. Let $\tau$ be a smooth anti-symplectic involution on $M$ compatible with the action of $T$. Suppose that $M^{T}=M^{T_{2}}$ and that $\pi_{0}\left(M^{T} \cap M^{\tau}\right) \rightarrow \pi_{0}\left(M^{T}\right)$ is a bijection. Then $M^{\tau}$ is $T_{2}$-equivariantly formal over $\mathbb{Z}_{2}$.

Proof As $\pi_{0}\left(M^{T} \cap M^{\tau}\right) \rightarrow \pi_{0}\left(M^{T}\right)$ is a bijection, by 8, Lemma 2.1 and Theorem 3.1], we know that $B\left(M^{\tau}\right)=B\left(M^{\tau} \cap M^{T}\right)$. By Lemma 8.1 $M^{\tau} \cap M^{T}=$ $\left(M^{\tau}\right)^{T_{2}}$ so $B\left(M^{\tau}\right)=B\left(\left(M^{\tau}\right)^{T_{2}}\right)$. This implies that $M^{\tau}$ is $T_{2}$-equivariantly formal over $\mathbb{Z}_{2}$ (see, e.g. [1, Proposition 1.3.14]). 


\subsection{The main theorems}

Theorem 8.3 Let $M$ be a compact symplectic manifold equipped with a Hamiltonian action of a torus $T$ and with a compatible smooth anti-symplectic involution $\tau$. If $M^{T}$ is a conjugation space, then $M$ is a conjugation space.

Proof Choose a generic $\xi \in \mathfrak{t}$ so that $\Phi^{\xi}: M \rightarrow \mathbb{R}$ is a Morse-Bott function with critical set $M^{T}$. Let $c_{0}<c_{1}<\cdots<c_{N}$ be the critical values of $\Phi^{\xi}$, and let $F_{i}=\left(\Phi^{\xi}\right)^{-1}\left(c_{i}\right) \cap M^{T}$ be the critical sets. Let $\varepsilon>0$ be less than any of the differences $c_{i}-c_{i-1}$, and define $M_{i}=\left(\Phi^{\xi}\right)^{-1}\left(\left(-\infty, c_{i}+\varepsilon\right]\right)$. We will prove by induction that $M_{i}$ is a conjugation space. This is true for $i=0$ since $M_{0}$ is $C$-homotopy equivalent to $F_{0}$, which is a conjugation space by hypothesis. By induction, suppose that $M_{i-1}$ is a conjugation space.

We saw in Subsection 8.1 that the negative normal bundle $\nu_{i}$ to $F_{i}$ is a $\tau$-bundle. The pair $\left(M_{i}, M_{i-1}\right)$ is $C$-homotopy equivalent to the pair $\left(\mathbb{D}\left(\nu_{i}\right), \mathbb{S}\left(\nu_{i}\right)\right)$. Since $F_{i}$ is a conjugation space by hypothesis, the pair $\left(M_{i}, M_{i-1}\right)$ is conjugation pair by Proposition 6.4. Therefore, $M_{i}$ is a conjugation space by Proposition 4.1 We have thus proven that each $M_{i}$ is a conjugation space, including $M_{N}=M$.

Remark 8.4 The proof of Theorem 8.3 shows that the compactness assumption on $M$ can be replaced by the assumptions that $M^{T}$ consists of finitely many connected components, and that some generic component of the moment map $\Phi: M \rightarrow \mathfrak{t}^{*}$ is proper and bounded below. That $M^{T}$ has finitely many connected components ensures that $H_{T}^{*}(M)$ is a finite rank module over $H_{T}^{*}(p t)$. That some component of the moment map is proper and bounded below ensures that that component of the moment map is a Morse-Bott function on $M$. Examples of this more general situation include hypertoric manifolds (see [12]).

Using Theorem 7.5] and Corollary [7.5, we get the following corollary of Theorem 8.3

Corollary 8.5 Let $M$ be a compact symplectic manifold equipped with a Hamiltonian action of a torus $T$ and a compatible smooth anti-symplectic involution $\tau$. If $M^{T}$ is a conjugation space, then $M_{T}$ is a conjugation space. In particular, there is a ring isomorphism

$$
\bar{\kappa}: H_{T}^{2 *}(M) \stackrel{\approx}{\longrightarrow} H_{T_{2}}^{*}\left(M^{\tau}\right) .
$$


Finally, the same proof as for Theorem 8.3 using Proposition 6.7 instead of Proposition 6.4 gives the following:

Theorem 8.6 Let $M$ be a compact symplectic manifold equipped with a Hamiltonian action of a torus $T$ and with a compatible smooth anti-symplectic involution $\tau$. If $M^{T}$ is a spherical conjugation complex, then $M$ is a spherical conjugation complex.

Examples 8.7 The theorems of this subsection apply to toric manifolds $\left(M^{T}\right.$ is discrete). They also apply to spatial polygon spaces $\operatorname{Pol}(a)$ of $m$ edges, with lengths $a=\left(a_{1}, \ldots, a_{m}\right)$ (see, e.g. [13]), the involution being given by a mirror reflection [13, §,9]. One proceeds by induction $m$ (for $m \leq 3, \operatorname{Pol}(a)$ is either empty or a point). The induction step uses that $\operatorname{Pol}(a)$ generically admits compatible Hamiltonian circle action, called bending flows, introduced by Klyachko (19, see, e.g. 14), for which the connected component of the fixed point set are polygon spaces with fewer edges [14, Lemma 2.3].

Therefore, toric manifolds and polygon spaces are spherical conjugation complexes. The isomorphism $\kappa$ were discovered in [7] and [13, $\S 9$ ].

\subsection{The Chevalley involution on co-adjoint orbits of semi-simple compact Lie groups}

The goal of this section is to show that coadjoint orbits of compact semi-simple Lie groups are equipped with a natural involution which makes them conjugation spaces. Let $\mathfrak{l}$ be a semi-simple complex Lie algebra, and $\mathfrak{h}$ a Cartan sub-algebra with roots $\Delta$. Multiplication by -1 on $\Delta$ induces, by the isomorphism theorem [23. Corollary $\mathrm{C}, \S 2.9]$, a Lie algebra involution $\sigma$ on $\mathfrak{l}$ called the Chevalley involution [23. Example p. 51]. Then $\sigma(h)=-h$ for $h \in \mathfrak{h}$ and $\sigma\left(X_{\alpha}\right)=-X_{-\alpha}$, if $X_{\alpha}$ is the weight vector occurring in a Chevalley normal form [23, Theorem A, $\S 2.9]$. By construction of the compact form $\mathfrak{l}_{0}$ of $\mathfrak{l}[23$, $\S 2.10]$, the involution $\sigma$ induces a Lie algebra involution on the real Lie algebra $\mathfrak{l}_{0}$, still called the Chevalley involution and denoted by $\sigma$. This shows that any semi-simple compact real Lie algebra admits a Chevalley involution. For instance, if $\mathfrak{l}=\mathfrak{s l}(n, \mathbb{C})$, then $\sigma(X)=-X^{T}$ and the induced Chevalley involution on $\mathfrak{l}_{0}=\mathfrak{s u}(n)$ is complex conjugation.

Let $G$ be a compact semi-simple Lie group with Lie algebra $\mathfrak{g}$ and a maximal torus $T$. Recall that the dual $\mathfrak{g}^{*}$ of $\mathfrak{g}$ is endowed with a Poisson structure characterized by the fact that $\mathfrak{g}^{* *}$ is a Lie sub-algebra of $\mathcal{C}^{\infty}(\mathfrak{g})$ and the 
canonical map $\mathfrak{g} \stackrel{\approx}{\underset{ }{\longrightarrow}} \mathfrak{g}^{* *}$ is a Lie algebra isomorphism. Therefore, the map $\tau=-\sigma^{*}: \mathfrak{g}^{*} \rightarrow \mathfrak{g}^{*}$ is an anti-Poisson involution, called again the Chevalley involution on $\mathfrak{g}^{*}$.

Theorem 8.8 The Chevalley involution $\tau$ preserves each coadjoint orbit $\mathcal{O}$, and induces an anti-symplectic involution $\tau: \mathcal{O} \rightarrow \mathcal{O}$ with respect to which $\mathcal{O}$ is a conjugation space. One also has an ring-isomorphism

$$
\bar{\kappa}: H_{T}^{2 *}(\mathcal{O}) \stackrel{\approx}{\longrightarrow} H_{T_{2}}^{*}\left(\mathcal{O}^{\tau}\right) .
$$

Proof The coadjoint orbits are the symplectic leaves of the Poisson structure on $\mathfrak{g}^{*}$. As $\tau$ is anti-Poisson, the image $\tau(\mathcal{O})$ of a coadjoint orbit $\mathcal{O}$ is also a coadjoint orbit $\mathcal{O}^{\prime}$. We will show that $\mathcal{O}^{\prime}=\mathcal{O}$. Since $G$ is semi-simple, the Killing form $\langle$,$\rangle is negative definite. Thus, the map K: \mathfrak{g} \rightarrow \mathfrak{g}^{*}$ given by $K(x)(-)=\langle x,-\rangle$ is an isomorphism. It intertwines the adjoint action with the coadjoint action and satisfies $\tau \circ K=-K \circ \sigma$.

Now, to show $\mathcal{O}^{\prime}=\mathcal{O}$, if $\mathcal{O}$ is a coadjoint orbit, the adjoint orbit $K^{-1}(\mathcal{O})$ contains an element $t \in \mathfrak{t}$. Thus, $\tau(K(t))=-K(\sigma(t))=K(t)$. Therefore $\mathcal{O}^{\prime}=$ $\tau(\mathcal{O})=\mathcal{O}$. As $\tau$ is anti-Poisson on $\mathfrak{g}^{*}$, its restriction to $\mathcal{O}$ is anti-symplectic. Moreover, since $\sigma$ is -1 on $\mathfrak{t}$, the involution $\tau$ is compatible with the coadjoint action of $T$ on $\mathcal{O}$. Finally, $\mathcal{O}^{T}$ is discrete, and $\mathcal{O} \cap K(\mathfrak{t})=\mathcal{O}^{T} \subset \mathcal{O}^{\tau}$. It is clear, then, that $\mathcal{O}^{T}$ is a conjugation space. The theorem now follows from Theorem 8.3 and Corollary 8.5 .

Remark 8.9 The conjugation cells used to build $\mathcal{O}$ as a conjugation space are precisely the Bruhat cells of the coadjoint orbit. The Bruhat decomposition is $\tau$-invariant.

In type $A$, the Chevalley involution is complex conjugation on $\mathfrak{s u}(n)$. In this case, Theorem 8.8 has been proven in 24] and 4]. In those papers, the authors use the fact that the isotropy weights at each fixed point are pairwise independent over $\mathbb{F}_{2}$. This condition is not satisfied in general for the coadjoint orbits of other types. Indeed, for the generic orbits, these weights are a set of positive roots and the other types have strings of roots of length at least 2 . This can be seen already in the moment polytopes for generic coadjoint orbits of $B_{2}$ and $G_{2}$, shown in Figure 8.1.

In [24] and [4], the isomorphism

$$
\bar{\kappa}: H_{T}^{2 *}(\mathcal{O}) \stackrel{\approx}{\longrightarrow} H_{T_{2}}^{*}\left(\mathcal{O}^{\tau}\right)
$$




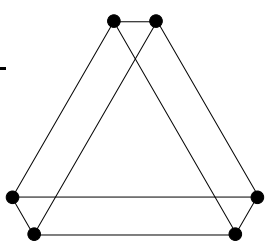

(a)

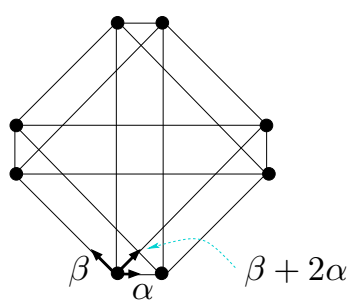

(b)

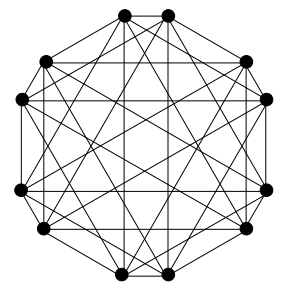

(c)

Figure 8.1: The moment polytopes for the generic coadjoint orbits of simple Lie groups of rank 2: we show types (a) $A_{2}$, (b) $B_{2}$ and (c) $G_{2}$. As shown in (b), for type $B_{2}$, at a $T$-fixed point, we can see that $\beta, \alpha$ and $\beta+2 \alpha$ are isotropy weights. There is a similar occurrence for type $G_{2}$.

is proved by giving a combinatorial description of each of these rings, and noting that these descriptions are identical. This combinatorial description does not generally apply in the other types precisely because the isotropy weights the fixed points are not pairwise independent over $\mathbb{F}_{2}$. Nevertheless, we still have the isomorphism on the equivariant cohomology rings.

\subsection{Symplectic reductions}

Let $M$ be a compact symplectic manifold equipped with a Hamiltonian action of a torus $T$ and a compatible smooth anti-symplectic involution $\tau$. We saw in Theorem 8.3 that if $M^{T}$ is a conjugation space, then $M$ is a conjugation space. Using this, we extend results of Goldin and the second author 9 to show that in certain cases, the symplectic reduction is again a conjugation space. To do this, we must construct a ring isomorphism

$$
\kappa_{\text {red }}: H^{2 *}(M / / T(\mu)) \rightarrow H^{*}\left((M / / T(\mu))^{\tau_{r e d}}\right),
$$

and a section

$$
\sigma_{\text {red }}: H^{2 *}(M / / T(\mu)) \rightarrow H_{C}^{2 *}(M / / T(\mu))
$$

that satisfy the conjugation equation.

Let $\Phi: M \rightarrow \mathfrak{t}^{*}$ be the moment map for $M$. When $\mu \in \mathfrak{t}^{*}$ is a regular value of $\Phi$, and when $T$ acts on $\Phi^{-1}(\mu)$ freely, we define the symplectic reduction

$$
M / / T(\mu)=\Phi^{-1}(\mu) / T \text {. }
$$


Kirwan [16] proved that the inclusion map $\Phi^{-1}(\mu) \hookrightarrow M$ induces a surjection in equivariant cohomology with rational coefficients:

$$
H_{T}^{*}(M ; \mathbb{Q}) \stackrel{\mathcal{K}}{\longrightarrow} H_{T}^{*}\left(\Phi^{-1}(\mu) ; \mathbb{Q}\right)=H^{*}(M / / T(\mu) ; \mathbb{Q}) .
$$

The map $\mathcal{K}$ is called the Kirwan map. Under additional assumptions on the torsion of the fixed point sets and the group action, this map is surjective over the integers or $\mathbb{Z}_{2}$ as well. There are several ways to compute the kernel of $\mathcal{K}$. Tolman and Weitsman [27] did so in the way that is most suited to our needs.

Goldin and the second author extend these two results to the real locus, when the the torus action has suitable 2-torsion.

Definition 8.10 Let $x \in M$, and suppose $H$ is the identity component of the stabilizer of $x$. Then we say $x$ is a 2 -torsion point if there is a weight $\alpha$ of the isotropy action of $H$ on the normal bundle $\nu_{x} M^{H}$ that satisfies $\alpha \equiv 0 \bmod 2$.

The necessary assumption is that $M^{\tau}$ have no 2 -torsion points. This hypothesis is reasonably strong. Real loci of toric varieties and coadjoint orbits in type $A_{n}$ satisfy this hypothesis, for example, but the real loci of maximal coadjoint orbits in type $B_{2}$ do not.

We now define reduction in the context of real loci. Fix $\mu$ a regular value of $\Phi$ satisfying the condition that $T$ acts freely on $\Phi^{-1}(\mu)$. Then $M_{\text {red }}=$ $M / / T(\mu)$ is again a symplectic manifold with a canonical symplectic form $\omega_{\text {red }}$. Moreover, there is an induced involution $\tau_{r e d}$ on $M_{r e d}$, and this involution is anti-symplectic. Thus, the fixed point set of this involution $(M / / T(\mu))^{\tau_{r e d}}$ is a Lagrangian submanifold of $M$. We now define

$$
M^{\tau} / / T_{2}(\mu):=\left(\left(\left.\Phi\right|_{M^{\tau}}\right)^{-1}(\mu)\right) / T_{2} .
$$

When $T$ acts freely on the level set, Goldin and the second author 9] show that

$$
(M / / T(\mu))^{\tau_{r e d}}=M^{\tau} / / T_{2}(\mu) .
$$

We can now start proving that, under certain hypotheses, the quotient $M / / T(\mu)$ is a conjugation space. We begin by constructing the isomorphism $\kappa_{\text {red }}$.

Proposition 8.11 Suppose $M$ is a compact symplectic manifold equipped with a Hamiltonian action of a torus $T$ and a compatible smooth anti-symplectic involution $\tau$. Suppose further that $M^{T}$ is a conjugation space, and that $M$ contains no 2 -torsion points. Then there is an isomorphism

$$
\kappa_{\text {red }}: H^{2 *}(M / / T(\mu)) \stackrel{\approx}{\longrightarrow} H^{*}\left(M^{\tau} / / T(\mu)\right)=H^{*}\left((M / / T(\mu))^{\tau_{\text {red }}}\right),
$$

induced by $\kappa$. 
Proof The first main theorem of [9] states that when $M^{\tau}$ contains no 2-torsion points, the real Kirwan map in equivariant cohomology

$$
\mathcal{K}^{\tau}: H_{T_{2}}^{*}\left(M^{\tau}\right) \rightarrow H_{T_{2}}^{*}\left(\left.\Phi\right|_{M^{\tau}} ^{-1}(\mu)\right)=H^{*}\left(M^{\tau} / / T(\mu)\right),
$$

induced by inclusion, is a surjection. The proof of surjectivity makes use of the function $\|\Phi-\mu\|^{2}$ as a Morse-Kirwan function on $M^{\tau}$. The critical sets of this function are possibly singular, but the hypothesis that the real locus have no 2-torsion points allows enough control over these critical sets to prove surjectivity.

Let $x \in M^{T}$. By assumption $x$ is not a 2-torsion point, so Condition (3) of Lemma 8.1 is satisfied. Lemma 8.1 then implies that $M^{T}=M^{T_{2}}$. We now show that there is a commutative diagram

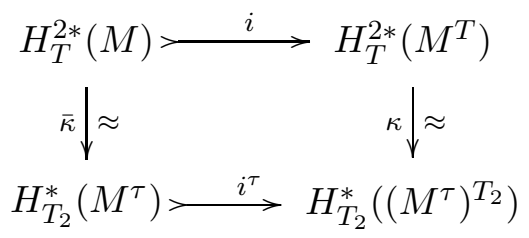

where the horizontal arrows are induced by inclusions. To see this, we first note that because $M^{T}$ is a conjugation space, then $M_{T}$ is a conjugation space by Corollary [8.5, which also gives the left isomorphism $\bar{\kappa}$. The trivial $T$ action on $M^{T}$ is also compatible with $\tau$. By Theorem [7.5 one have a ring isomorphism $\kappa: H_{T}^{2 *}\left(M^{T}\right) \stackrel{\approx}{\longrightarrow} H_{T_{2}}^{*}\left(M^{\tau} \cap M^{T}\right)$. As $M^{T}=M^{T_{2}}$, we deduce that $M^{\tau} \cap M^{T}=\left(M^{\tau}\right)^{T_{2}}$ by Lemma 8.1, whence the the right vertical isomorphism $\kappa$.

Diagram 8.5 is commutative by the naturality of $H^{*}$-frames (Proposition 3.14). Finally, $M^{\tau}$ is $T_{2}$-equivariantly formal over $\mathbb{Z}_{2}$ by Lemma 8.2. Therefore $i^{\tau}$ is injective by, e.g. 1, Proposition 1.3.14]. It follows that $i$ is also injective.

Note that Kirwan showed that $i$ is injective when the coefficient ring is $\mathbb{Q}$. However, an additional assumption on $M^{T}$ is needed to extend her proof to the coefficient ring $\mathbb{Z}_{2}$, so we may not conclude that directly.

We denote the restriction of a class $\alpha \in H_{T}^{*}\left(M^{\tau}\right)$ to the fixed points by $\left.\alpha\right|_{\left(M^{\tau}\right)^{T_{2}}} \in H_{T_{2}}^{*}\left(\left(M^{\tau}\right)^{T_{2}}\right)$. The second main result of [9] computes the kernel of $\mathcal{K}^{\tau}$. For every $\xi \in \mathfrak{t}$, let

$$
M_{\xi}^{\tau}=\left\{p \in M^{\tau} \mid\langle\Phi(p), \xi\rangle \leq 0\right\} \subseteq M^{\tau} .
$$

Let $F=M^{T}$ denote the fixed point set, and let

$$
K_{\xi}^{\tau}=\left\{\alpha \in H_{T}^{*}\left(M^{\tau}\right)|\alpha|_{F \cap M_{\xi}^{\tau}}=0\right\}
$$


Finally, let $K^{\tau}$ be the ideal generated by the ideals $K_{\xi}^{\tau}$ for all $\xi \in \mathfrak{t}$. Then there is a short exact sequence, in cohomology with $\mathbb{Z}_{2}$ coefficients,

$$
0 \rightarrow K^{\tau} \rightarrow H_{T}^{*}\left(M^{\tau}\right) \rightarrow H^{*}\left(M^{\tau} / / T(\mu)\right) \rightarrow 0 .
$$

The important thing to notice is that this description of the kernel is identical to the description of the kernel for $M$, given by Tolman and Weitsman, when $M$ contains no 2-torsion points. The fact that Diagram 8.5 commutes implies that the support of a class $\kappa(\alpha)$ is the real locus of the support of $\alpha$. Therefore, there is a natural isomorphism between $K$ and $K^{\tau}$ induced by $\kappa$. Thus, we have a commutative diagram:

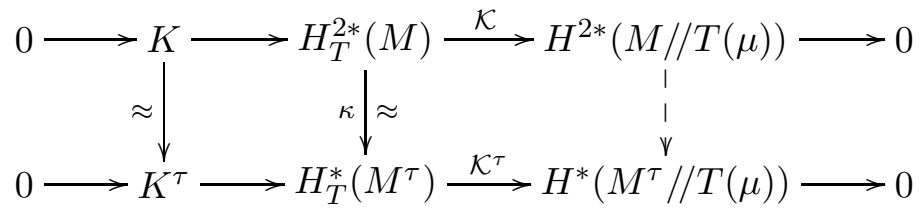

Therefore, the vertical dashed arrow represents an induced isomorphism

$$
\kappa_{\text {red }}: H^{2 *}(M / / T(\mu)) \stackrel{\approx}{\longrightarrow} H^{*}\left(M^{\tau} / / T(\mu)\right),
$$

as rings.

Now that we have established the isomorphism $\kappa_{\text {red }}$ between the cohomology of the symplectic reduction and the cohomology of its real points, we must find the map $\sigma_{\text {red }}$ and prove the conjugation relation. We have the following commutative diagram:

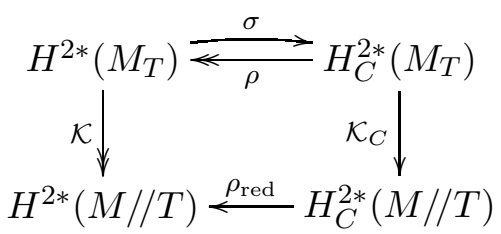

As the diagram commutes, we see that $\rho_{\text {red }}$ is a surjection. Moreover, because $\mathcal{K}$ is a surjection, we may choose an additive section $s: H^{2 *}(M / / T) \rightarrow H^{2 *}\left(M_{T}\right)$ and then define a section $\sigma_{\text {red }}:=\mathcal{K}_{C} \circ \sigma \circ s$ of $\rho_{\text {red }}$. Adding the restriction maps into the diagram, we have:

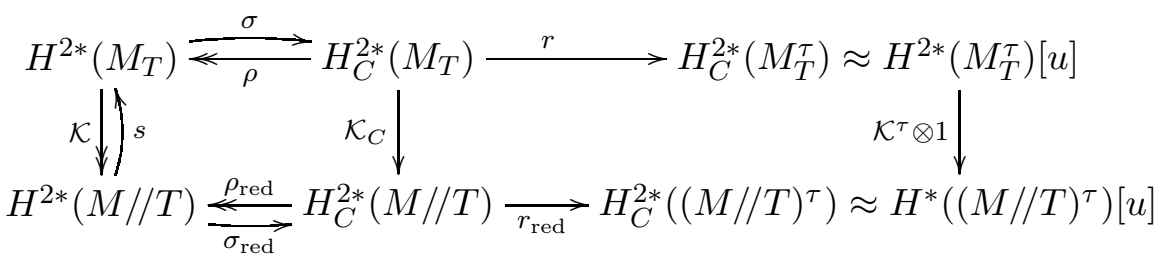


Now we check, for $a \in H^{2 m}(M / / T)$,

$$
\begin{aligned}
r_{\text {red }}\left(\sigma_{\text {red }}(a)\right) & =r_{\text {red }}\left(\mathcal{K}_{C} \circ \sigma \circ s(a)\right) \\
& =\mathcal{K}^{\tau} \otimes 1(r(\sigma(s(a)))) \\
& =\mathcal{K}^{\tau} \otimes 1\left(\kappa(s(a)) u^{m}+\ell t_{m}\right) \\
& =\kappa_{\text {red }}(a) u^{m}+\ell t_{m} .
\end{aligned}
$$

Thus, by the commutativity of diagram (8.7), we have proved the conjugation equation, and hence the following theorem.

Theorem 8.12 Let $M$ be compact symplectic manifold equipped with a Hamiltonian action of a torus $T$, with moment map $\Phi$, and with a compatible smooth anti-symplectic involution $\tau$. Suppose that $M^{T}$ is a conjugation space and that $M$ contains no 2 -torsion points. Let $\mu$ be a regular value of $\Phi$ such that $T$ acts freely on $\Phi^{-1}(\mu)$. Then, $M / / T(\mu)$ is a conjugation space.

Remark 8.13 When $T=S^{1}$ in Theorem 8.12 the symplectic cuts $C_{ \pm}$at $\mu$ introduced by E. Lerman [17] also inherit an Hamiltonian $S^{1}$-action and a compatible anti-symplectic involution. The connected components of $C_{ \pm}^{T}$ are those of $M^{T}$ plus a copy of $M / / T(\mu)$. By Theorem 8.12, $C_{ \pm}^{T}$ are conjugation spaces. Therefore, using Theorems 8.3 and Corollary 8.5] we deduce that $C_{ \pm}$ and $\left(C_{ \pm}\right)_{T}$ are conjugation spaces.

\section{References}

[1] C Allday, V Puppe, Cohomological methods in transformation groups, Cambridge Studies in Advanced Mathematics 32, Cambridge University Press, Cambridge (1993) MathReview

[2] M F Atiyah, K-theory and reality, Quart. J. Math. Oxford Ser. (2) 17 (1966) 367-386 MathReview

[3] M Audin, The topology of torus actions on symplectic manifolds, Progress in Mathematics 93, Birkhäuser Verlag, Basel (1991) MathReview

[4] D Biss, V W Guillemin, T S Holm, The mod 2 cohomology of fixed point sets of anti-symplectic involutions, Adv. Math. 185 (2004) 370-399 MathReview

[5] A Borel, Seminar on transformation groups, with contributions by G. Bredon, EE Floyd, D Montgomery, R Palais, Annals of Mathematics Studies 46, Princeton University Press, Princeton, N.J. (1960) MathReview

[6] A Borel, A Haefliger, La classe d'homologie fondamentale d'un espace analytique, Bull. Soc. Math. France 89 (1961) 461-513 MathReview 
[7] M W Davis, T Januszkiewicz, Convex polytopes, Coxeter orbifolds and torus actions, Duke Math. J. 62 (1991) 417-451 MathReview

[8] J J Duistermaat, Convexity and tightness for restrictions of Hamiltonian functions to fixed point sets of an antisymplectic involution, Trans. Amer. Math. Soc. 275 (1983) 417-429 MathReview

[9] R F Goldin, T S Holm, Real loci of symplectic reductions, Trans. Amer. Math. Soc. 356 (2004) 4623-4642 MathReview

[10] M Goresky, R Kottwitz, R MacPherson, Equivariant cohomology, Koszul duality, and the localization theorem, Invent. Math. 131 (1998) 25-83 MathReview

[11] M J Greenberg, J R Harper, Algebraic topology, Mathematics Lecture Note Series 58, Benjamin/Cummings Publishing Co. Inc. Advanced Book Program, Reading, Mass. (1981) MathReview

[12] T Holm, M Harada, The equivariant cohomology of hypertoric varieties and their real loci, Commun. Anal. Geom. 13 (2005) 645-677

[13] J-C Hausmann, A Knutson, The cohomology ring of polygon spaces, Ann. Inst. Fourier (Grenoble) 48 (1998) 281-321 MathReview

[14] J-C Hausmann, S Tolman, Maximal Hamiltonian tori for polygon spaces, Ann. Inst. Fourier (Grenoble) 53 (2003) 1925-1939 MathReview

[15] D Husemoller, Fibre bundles, Springer-Verlag, New York (1975) MathReview

[16] F C Kirwan, Cohomology of quotients in symplectic and algebraic geometry, Mathematical Notes 31, Princeton University Press, Princeton, NJ (1984) MathReview

[17] E Lerman, Symplectic cuts, Math. Res. Lett. 2 (1995) 247-258 MathReview

[18] A T Lundell, S Weingram, The topology of $C W$-complexes Van Nostrand (1969)

[19] A A Klyachko, Spatial polygons and stable configurations of points in the projective line, from: "Algebraic geometry and its applications (Yaroslavl', 1992)", Aspects Math. E25, Vieweg, Braunschweig (1994) 67-84 MathReview

[20] J McCleary, A user's guide to spectral sequences, second edition, Cambridge Studies in Advanced Mathematics 58, Cambridge University Press (2001) MathReview

[21] J W Milnor, J D Stasheff, Characteristic classes, Princeton University Press, Princeton, NJ (1974) MathReview

[22] L O'Shea, R Sjamaar, Moment maps and Riemannian symmetric pairs, Math. Ann. 317 (2000) 415-457 MathReview

[23] H Samelson, Notes on Lie algebras, Universitext, Springer-Verlag, New York (1990) MathReview

[24] C Schmid, Cohomologie équivariante de certaines variétés hamiltoniennes et de leur partie réelle, thesis, University of Geneva (2001) 
[25] E H Spanier, Algebraic topology, McGraw-Hill Book Co. New York (1966) MathReview

[26] T tom Dieck, Transformation groups, de Gruyter Studies in Mathematics 8, Walter de Gruyter \& Co. Berlin (1987) MathReview

[27] S Tolman, J Weitsman, The cohomology rings of symplectic quotients, Comm. Anal. Geom. 11 (2003) 751-773 MathReview

[28] J A van Hamel, Algebraic cycles and topology of real algebraic varieties, CWI Tract 129, Stichting Mathematisch Centrum Centrum voor Wiskunde en Informatica, Amsterdam (2000) MathReview

[29] J A van Hamel, Personal correspondence, (March 2004)

Section de mathématiques, 2-4, rue du Lièvre

CP 64 CH-1211 Genève 4, Switzerland

Department of Mathematics, University of Connecticut

Storrs CT 06269-3009, USA

Universität Konstanz, Fakultät für Mathematik

Fach D202, D-78457 Konstanz, Germany

Email: hausmann@math.unige.ch, tsh@math.uconn.edu,

Volker.Puppe@uni-konstanz.de

Received: 16 February 2005 